\title{
IDENTIFICACIÓN DE LOS MÁXIMOS EVENTOS DE INUNDACIÓN MARINA MIOCENOS Y SU USO EN LA CORRELACIÓN Y ANÁLISIS DE LA CUENCA DE ANTEPAÍS DE LOS LLANOS ORIENTALES, COLOMBIA
}

Edward Duarte $^{1 *}$; German Bayona ${ }^{1}$; Carlos Jaramillo ${ }^{1,2}$; Mauricio Parra ${ }^{1,3}$ Ingrid Romero $^{2}$; Josué Alejandro Mora ${ }^{4}$ DOI: http://dx.doi.org/10.18273/revbol.v39n1-2017001 @) (1)

Forma de citar: Duarte, E., Bayona, G., Jaramillo, C., Parra, M., Romero, I., y Mora, J.A. 2017. Identificación de los máximos eventos de inundación marina Miocenos y su uso en la correlación y análisis de la cuenca de antepaís de los Llanos Orientales, Colombia. Boletín de Geología, 39(1): 19-40.

\section{RESUMEN}

Los máximos eventos de inundación marina en una cuenca antepaís con relleno dominantemente continental, como la cuenca de los Llanos de Colombia, son superficies de correlación cronoestratigráfica de carácter regional. A pesar de las implicaciones de estas superficies en la prospectividad de hidrocarburos, no se conoce la extensión lateral, duración e implicaciones en la evolución de la cuenca correspondiente a los eventos de inundación marina del Mioceno. El análisis de estratigrafía secuencial a partir de información de superficie, pozos, sísmica y palinología, permite identificar dos eventos de inundación marina en el Mioceno temprano y medio, un evento de inundación en el Oligoceno y dos límites de secuencia. Las dos superficies de máxima inundación marina del Mioceno son de carácter sincrónico y cubren completamente el sistema de acumulación de la cuenca de antepaís. La superficie de máxima inundación marina del Mioceno temprano se registra en los depósitos lodosos del miembro informal C2 de la Formación Carbonera, mientras la superficie de máxima inundación marina del Mioceno medio se registra en los depósitos lodosos de la Formación León. Adicionalmente se identificaron dos límites de secuencia hacia la parte media y tope de la Formación Carbonera.

Los mapas de espesores del Mioceno temprano y medio para la cuenca Llanos documentan la ubicación de los depocentros adyacente al frente de deformación. Estos depocentros delimitan zonas de mayor espesor e implican una carga de enterramiento para la roca fuente de Cretácico y Paleógeno durante el Mioceno medio y tardío. En el sector distal de los Llanos, sin embargo, la tendencia de los espesores cambia entre el Mioceno temprano y medio. Durante el Mioceno temprano el espesor incrementa hacia el norte, para el Mioceno medio el espesor incrementa hacia el sur. Para el sector distal de la cuenca la tasa de acumulación del Mioceno medio $(108 \mathrm{~m} / \mathrm{my}$.) es más del doble que la calculada para el Mioceno temprano $(47 \mathrm{~m} / \mathrm{my}$.). Este fuerte incremento en subsidencia total, se asocia al incremento en el acortamiento del flanco oriental de la Cordillera Oriental, levantamientos intracuenca relacionados al Arco de Vaupés y aumento global en el nivel base que favorece el aporte continuo de sedimentos y la generación de espacio de acomodación, pero no afecta la continua acumulación lodosa en los intervalos de inundación marina.

Palabras clave: Superficies de correlación, Eventos de Inundación, Mioceno, cuenca Llanos.

\section{IDENTIFICATION OF MIOCENE MAXIMUM MARINE FLOODING EVENTS \\ AND THEIR USE FOR CORRELATION AND ANALYSIS OF THE EASTERN LLANOS FORELAND BASIN, COLOMBIA}

\begin{abstract}
Maximum marine flooding events constitute regional chronostratigraphic correlative surfaces in foreland basins dominated by continental sedimentary fill, such as in the Colombian Llanos Basin. Despite of the importance of these surfaces for the hydrocarbon prospectivity, yet their lateral extent, duration, and basin evolution implications remain unknown. Analysis of sequence stratigraphy using outcrops, wells, seismic and palynological data, allowed the identification of two marine flooding events in the early and middle Miocene, and a third flooding event in the Oligocene, along with two sequence boundaries. The two Miocene flooding surfaces are synchronous and cover entirely this forelandbasin accumulation system. The early Miocene surface is recorded by muddy deposits of the informal member $\mathrm{C} 2$ of the Carbonera Formation, whereas the middle Eocene surface is recorded by the muddy deposits of the Leon Formation. Also, sequence boundaries were identified at the middle and top of the Carbonera Formation.

Thickness maps of early and middle Miocene for the entire Llanos Basin document the location of depocenters adjacent to the deformation front, which delineate thicker areas involving significant loading and more burial for the Cretaceous and Paleogene petroleum source rocks. However, more distally into the Llanos basin, thickness trends vary between the early and middle Miocene, revealing thickness increases towards the north during the early Miocene, and then increasing towards the south during middle Miocene. Accumulation rates of the middle Miocene (108 $\mathrm{m} /$ my) is more than twice than the calculated for the Early Miocene $(47 \mathrm{~m} / \mathrm{my})$, this strong increase represented in total subsidence is associated with an increase in shortening of the eastern flank of the Cordillera Oriental, intra-basinal uplifts related to the Vaupes arch and a global rise in the sea level, which favored the continuous sediment supply and the generation of accommodation space without affecting the continuous muddy accumulation during marine flooding events.
\end{abstract}

Keywords: Correlation surfaces, Flooding Events, Miocene, Llanos Basin.

${ }^{1}$ Corporación Geológica ARES, Bogotá, Colombia. (*) eduarte@cgares.org; gbayona@cgares.org

${ }^{2}$ Smithsonian Tropical Research Institute, Balboa, Ancon, Panama. JaramilloC@si.edu; incaromero@gmail.com

${ }^{3}$ Instituto de Energia e Ambiente, Universidade de São Paulo, São Paulo, Brasil. mparra@iee.usp.br

${ }^{4}$ HOCOL S.A, Bogotá, Colombia. Alejandro.mora@hocol.com.co 


\section{INTRODUCCIÓN}

Las cuencas sub-andinas que bordean el sector oriental de los Andes son de tipo antepaís y representan un registro estratigráfico muy completo de la historia de denudación de los cinturones montañosos adyacentes (Roddaz et al., 2010). Las cuencas de antepaís son definidas como una región elongada que se forma entre un cinturón orogénico y un cratón estable, principalmente en respuesta a la subsidencia flexural y levantamientos locales que genera la carga orogénica (Beaumont, 1981; DeCelles and Giles, 1996). El sistema antepaís se divide en provincias flexurales, las cuales sufren subsidencia (foredeep y backbulge) y levantamiento (forebulge) (Catuneanu, 2004; DeCelles and Giles, 1996). Debido a su relleno de carácter dominantemente continental en la cuenca de antepaís de los Andes del Norte (ver Roddaz et al. 2010 compilación), los cambios laterales en la magnitud de subsidencia flexural, aporte de sedimentos y la identificación de superficies estratigráficas con valor cronológico son factores críticos para entender la evolución del relleno de la cuenca. La identificación de las superficies de máxima inundación marina y los limites de secuencia han sido usados ampliamente como superficies de correlación desde mediados de los 90s en diferentes cuencas tipo antepaís alrededor del mundo (Giles and Dickinson, 1995; Pemberton and MacEachern, 1995; Schwans, 1995) incluyendo los Llanos de Colombia (Cooper et al., 1995).

En la cuenca de los Llanos (FIGURA 1), las rocas del Cenozoico registran el crecimiento del Orógeno de la Cordillera Oriental desde finales del Eoceno (Bayona et al., 2008a; Mora et al., 2010; Parra et al., 2009a). Este relleno consiste principalmente de rocas siliciclásticas procedentes de la erosión de la Cordillera Oriental al occidente y del escudo de la Guyana al oriente (Bayona et al., 2009b) con un eje deposicional que migra en la cuenca (Campos and Mann, 2015; Reyes-Harker et al., 2015). Transgresiones marinas regionales interrumpen la sedimentación continental y marginal de los Llanos (Boonstra et al., 2015). Las ingresiones marinas se identifican por sus litologías finas, amplia extensión regional, contenido de material biológico (fauna y flora) y un patrón de apilamiento uniforme. Estas transgresiones representan una superficie de correlación temporal efectiva, debido a su carácter sincrónico producto de su corta duración y amplia extensión.

En la exploración de hidrocarburos, la definición de la extensión y continuidad lateral de estas ingresiones marinas tiene implicaciones en la prospectividad. Debido a su amplia distribución a lo largo de la cuenca de los Llanos, estas litologías finas sirven como sello regional para la migración de hidrocarburos y aguas subterráneas (Cooper et al., 1995). Los reservorios clásticos en la cuenca de los Llanos incluyen depósitos de canales fluviales del Eoceno superior al Oligoceno (Cooper et al., 1995; Villamil, 2003). Recientemente se ha considerado que trampas estratigráficas producto del sello ocasionado por estos niveles de inundación pueden ser importantes y aún poco exploradas (Torrado et al., 2014). Estas acumulaciones de grano fino y origen marino también pueden presentar interés en los sistemas no convencionales de hidrocarburos. A pesar de su potencial relevancia como superficies de correlación en una cuenca de alta actividad exploratoria de hidrocarburos, no se ha documentado con detalle la extensión regional, espesor y temporalidad de las incursiones marinas y su carácter de correlación regional a lo largo de la cuenca de los Llanos Orientales.

Este estudio realiza un análisis estratigráfico secuencial que integra columnas estratigráficas en superficie, pozos, sísmica y bioestratigrafía, lo cual permite identificar las superficies de correlación cronoestratigráficas asociadas a estas incursiones marinas, con énfasis en los miembros informales de la Formación Carbonera y en la Formación León. Las superficies de máxima inundación marina son difíciles de identificar utilizando únicamente análisis de litofacies cuando las litologías son finas y homogéneas, y se requiere el uso de medidas palinológicas (i.e., índice de salinidad) que permiten identificar posibles incursiones marinas (Montaño et al., 2016; Santos et al., 2008). Finalmente, mapas de isócoros y mapas de tasas de acumulación de la Formación León y del intervalo superior de la Formación Carbonera con registro de ingresión marina permiten determinar si los mecanismos de generación de espacio de acomodación y aporte de sedimentos en la cuenca de los Llanos son constantes durante el Mioceno temprano y medio.

\section{GEOLOGÍA REGIONAL}

La cuenca de los Llanos corresponde a una cuenca antepaís desde finales del Eoceno tardío, la cual presenta altas tasas de acumulación y gran subsidencia hacia el sector del piedemonte (Bayona et al., 2008a; Cooper et al., 1995; Parra et al., 2009a; Parra et al., 2009b). El espesor de la sucesión Eoceno superior-presente varía de 4500 metros en el sector proximal hasta 600 metros en el sector distal de los Llanos Orientales (Bayona et al., 2008a; Bayona et al., 2008b; Sarmiento, 2011) у en donde las acumulaciones del Mioceno se extienden cerca de $500 \mathrm{~km}$ desde el frente de deformación hasta el escudo de Guyana (FIGURA 1) (Bayona et al., 2009a; Bayona et al., 2009b; Reyes-Harker et al., 2015). 


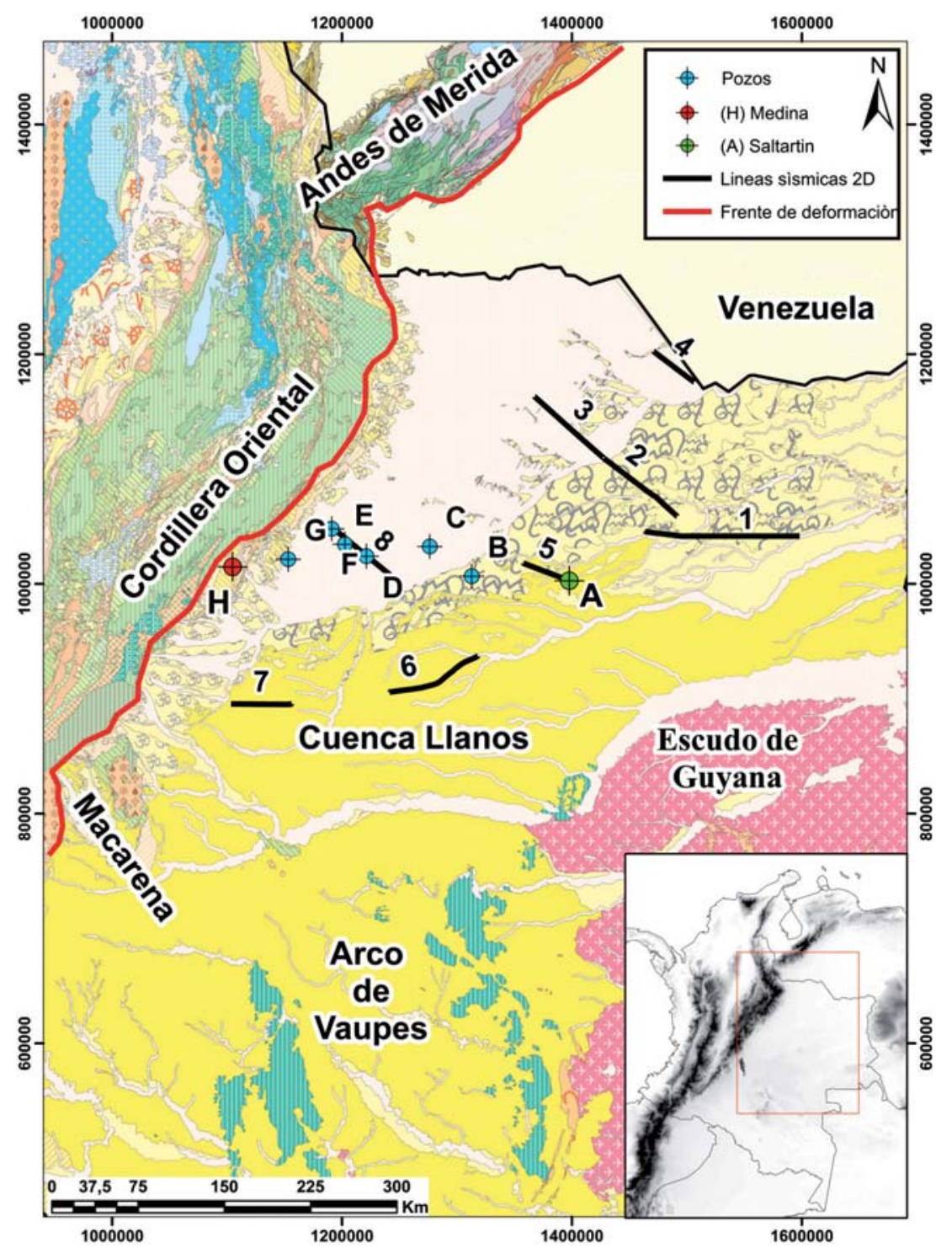

FIGURA 1. Mapa insertado: localización del área de estudio correspondiente a la cuenca de los Llanos, Colombia. Figura principal: mapa geológico de Colombia (Gomez et al., 2015) y mapa geológico de Venezuela (Hackley et al., 2006) en donde se muestra la localización de: pozo con núcleo (A) Saltarin 1A, 6 pozos $(\mathbf{B}-\mathbf{G})$ con información de registros eléctricos, 1 sección estratigráfica $(\mathbf{H})$ Medina y 8 líneas sísmicas 2D $(1-8)$ utilizadas en el presente estudio (Coordenadas geográficas en las Tablas 1 y 2).

Pocos estudios publicados se han enfocado sobre la estratigrafía del Mioceno y sus sistemas deposicionales (Parra et al., 2010), debido a que la historia exploratoria de hidrocarburos se concentra en estratos más antiguos. Estudios regionales (Campos and Mann, 2015; Cooper et al., 1995; Hermeston and Nemcok, 2013; MorenoLópez and Escalona, 2015; Sarmiento, 2011), entre otros, realizaron análisis tectono-estratigráficos secuenciales y estratigráficos a partir de facies sísmicas y registros de pozos. Estos estudios identificaron superficies de correlación regional en la cuenca Llanos, las cuales corresponden en algunos casos a topes formacionales (FIGURA 2), pero con pobre a nulo control bioestratigráfico. Estas superficies de correlación estratigráfica regional están controladas por: (1) la subsidencia tectónica regional (flexural y topografía dinámica) (Bayona et al., 2009a; Bayona et al., 2009b; Mora et al., 2015) (2) cambios laterales de espesor asociados al fallamiento normal como lo evidencia la deposición de la Formación León (Delgado et al., 2012), (3) fluctuaciones globales del nivel base asociados a la eustasia (Hardenbol et al., 1998), y (4) aporte de sedimentos desde las áreas fuentes (Bayona et al., 2007; Campos and Mann, 2015; Roddaz et al., 
2010). Por consiguiente, es necesario corroborar con la bioestratigrafía si estas superficies identificadas tienen validez como superficies cronoestratigráficas.

Dos secuencias tectono-estratigráficas del Eoceno tardío al reciente han sido identificadas para la cuenca de los Llanos (Bayona et al., 2008a; Campos and Mann, 2015; Cooper et al., 1995; Moreno-López and Escalona, 2015). Estratos proximales al foredeep de edad Oligoceno - Mioceno temprano, pertenecientes a la Formación Carbonera, fueron acumuladas durante el crecimiento de estructuras a lo largo del flanco oriental de la Cordillera Oriental (Bayona et al., 2008a; Parra et al., 2009a). Esta secuencia tectono-estratigráfica se caracteriza por presentar alternancia entre depósitos lodosos y arenosos, con depósitos conglomeraticos localizados cerca a las fallas inversas activas (Parra et al., 2010) y una geometría en cuña de los depósitos de antepaís que engrosan hacia el frente de deformación. Los estratos de grano fino incluyen lodolitas laminadas con moluscos e indicadores de acumulación continental (Bayona et al., 2008b; Parra et al., 2009a). Estos depósitos se acumularon en planicies fluvio-deltaicos desarrollados en el sector proximal del foredeep, pasando a una llanura costera y a un sistema lacustrelagunar en el sector central del foredeep, y a un sistema dominantemente fluvial derivado del escudo de la Guyana en el sector distal del foredeep (Bayona et al., 2008a; Bayona et al., 2007; Bayona et al., 2008b; Reyes-Harker et al., 2015). Una inundación marina de corta duración en el Mioceno temprano ya ha sido documentada hacia el piedemonte de los Llanos (Gomez et al., 2009) y en unidades más antiguas al Mioceno en otros sectores de los Llanos (Bayona et al., 2007).

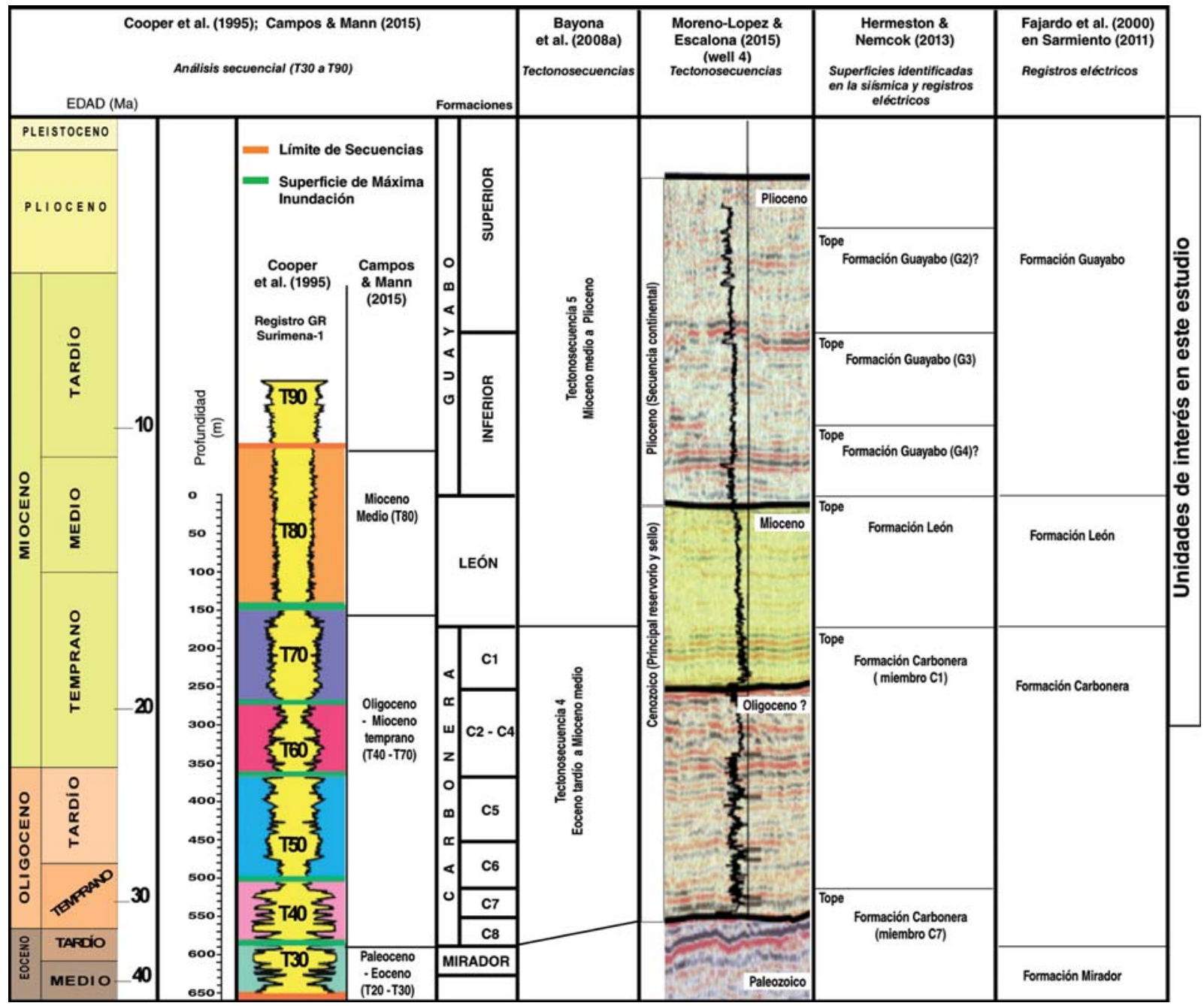

FIGURA 2. Gráfico de comparación de los principales estudios realizados del Eoceno tardío al presente en la cuenca de los Llanos Orientales donde se identificaron las principales superficies de correlación o límites de formaciones determinadas a partir de diferentes tipos de análisis. 
Una segunda tectono-secuencia, de edad Mioceno medio al reciente, consiste en su intervalo más inferior, de lodolitas laminadas y shales con registros aislados de moluscos y foraminíferos correspondiente a la Formación León (Mioceno medio); mientras que el contenido arenoso se incrementa hacia el norte y al occidente (Cooper et al., 1995). La Formación Guayabo (Mioceno superior-Plioceno) suprayace esta unidad, y consiste en depósitos grano-crecientes con mayor espesor hacia el frente de deformación andina (Parra et al., 2009a; Parra et al., 2010). Esta unidad se acumuló en ambientes netamente fluviales a aluviales derivados de la Cordillera Oriental hacia el este y el Arco de Vaupés hacia el sur (Bayona et al., 2008a; Bayona et al., 2008b; Mora et al., 2010; Reyes-Harker et al., 2015). Análisis palinológicos han indicado que la cuenca fue inundada por sistemas de aguas frescas con incursiones menores de aguas salobres (Bayona et al., 2008b; Hoorn et al., 1996). Estas inundaciones podrían estar relacionadas con ascensos eustáticos y tendrían una escala continental, conectando el caribe Venezolano con los Llanos Orientales y el sector noroccidental Amazónico (Hoorn et al., 1995; Hoorn et al., 1996).

\section{MÉTODOS}

\section{Análisis estratigráfico secuencial en afloramiento y pozos}

Para el control sedimentológico y estratigráfico de las unidades del Mioceno se consideraron: (1) los análisis sedimentológicos, palinológicos y petrográficos de la secuencia Carbonera C3 a Guayabo del pozo SALTARIN 1A, en el sector distal de la cuenca Llanos es tomado de Bayona et al. (2008b) y (Jaramillo et al., manuscript in review); y (2) la descripción estratigráfica e interpretación de ambientes sedimentarios de la secuencia Carbonera C5 a Guayabo en la sección de Medina, es tomada de Parra et al. (2010).

De estos dos estudios se identificaron tendencias de tamaño de grano, estructuras sedimentarias, composición de clastos, espesores de capas, bioperturbación y presencia de macrofósiles, lo cual permite identificar litofacies.Las asociaciones de litofacies dentro de una asociación vertical son tomadas como base de la interpretación de los sistemas depositacionales dominantes entre superficies estratigráficas.

Para refinar la interpretación de ambientes sedimentarios de estos dos estudios, se integra los análisis bioestratigráficos de 138 muestras en SALTARIN 1A (Jaramillo et al., en revisión; Romero, 2014) y 314 muestras en la sección de Medina (Jaramillo et al., 2011). Los resultados obtenidos por el análisis bioestratigráfico permiten: (1) determinar la temporalidad de las incursiones marinas, (2) refinar la datación de las unidades de interés (la zonación bioestratigráfica utilizada sigue la zonación palinológica para el Cenozoico de los Llanos y el piedemonte presentada en Jaramillo et al. (2011), y (3) realizar un análisis estratigráfico secuencial siguiendo los criterios descritos en Catuneanu et al. (2009) para la identificación de secuencias estratigráficas menores a 1 my de duración (secuencias estratigráficas de cuarto orden). Este último procedimiento permite la identificación de las siguientes superficies de correlación: límites de secuencias (superficies de máxima progradación) y superficies máximas de inundación. Adicionalmente se establece los intervalos de incursión marina (que pueden incluir una o más secuencias) donde la acumulación marina predominó sobre la continental a marginal.

Seis pozos (TABLA 1) profundos fueron seleccionados para realizar la correlación de las superficies estratigráficas identificadas en el piedemonte y el pozo SALTARIN 1A (información suministrada por la ANH). La interpretación estratigráfica secuencial de estos pozos tiene en cuenta la respuesta de electrofacies (tendencia entre geometrías de campana, cilíndrica y ovalada) en los perfiles Gamma Ray (GR), y el control de edad y reporte de ensamble palinológico de agua salobre determinados en análisis palinológicos en ripios reportados en Bayona et al. (2007). Los intervalos de incursión marina definidos en pozos son inferidos, ya que estos intervalos deben tener un control sistemático, preferiblemente en núcleos. Estos pozos son utilizados para determinar las tendencias depositacionales dentro de un régimen sintectónico de deposición, como se describe en Van Wagoner (1995).

\section{Análisis palinológicos}

El pozo Saltarin y la seccion de Medina fueron analizados palinológicamente con el objetivo de determinar: (1) El tope de la zona palinológica cuya biozonación sigue a Jaramillo et al. (2011). (2) Calcular el porcentaje de índice de salinidad (\%IS) que consiste en la proporción de palinomorfos y foraminiferos quitinosos marinos en relación al conteo total de cada muestra. Para cada muestra se contaron al menos 200 palinomorfos y foraminiferos quitinosos, los cuales se clasificaron en tres categorías: terrestres (polen y esporas), marinos (dinoflagelados, microforaminiferos, acritarcos marinos) y lacustrinos (Pediastrum y Botryococcus). Muestras con menos de 150 granos no fueron incluidas en el análisis. 
Las muestras fueron procesadas por el laboratorio Palinoflora (Bucaramanga, Colombia) que sigue la técnica standard palinológica (Traverse, 2007) pero no usa centrifugación, ya que esta tiende a destruir los dinoflagelados y foraminíferos quitinosos. Para mayor detalle del método utilizado, ver Santos et al. (2008).

TABLA 1. Coordenadas geográficas e información de pozos, núcleo y afloramiento (sistema de coordenadas proyectadas MAGNA Colombia Bogotá) usados en el presente estudio.

\begin{tabular}{ccccccc}
\hline \multirow{2}{*}{ ID } & \multirow{2}{*}{ NOMBRE } & \multicolumn{2}{c}{ COORDENADAS } & \multirow{2}{*}{ REFERENCIA } & \multirow{2}{*}{ TIPO } \\
\cline { 3 - 4 } & & $\mathbf{X}$ & $\mathbf{Y}$ & & núcleo \\
A & SALTARIN 1A & 1397725 & 1002792 & & Bayona et al., 2008b & pozo \\
B & CABI0001 & 1312816 & 1006726 & & Bayona et al., 2007 & pozo \\
C & GUAQ0001 & 1276316 & 1032627 & & Bayona et al., 2007 & pozo \\
D & LPUN0001 & 1221200 & 1024210 & & Bayona et al., 2007 & pozo \\
E & CENT0001 & 1202560 & 1034605 & & Bayona et al., 2007 & pozo \\
F & LGLO0001 & 1191542 & 1049605 & & Bayona et al., 2007 & pozo \\
G & FORA0001A & 1153248 & 1021528 & & Bayona et al., 2007 & afloramiento \\
H & MEDINA & 1104351 & 1014422 & & Parra et al., 2010 &
\end{tabular}

\section{Análisis de información sísmica}

La extensión lateral que presentan los intervalos de incursión marina, desde el sector distal hasta el sector proximal de la cuenca Llanos se determinó a partir de la interpretación de $470 \mathrm{~km}$ de líneas sísmicas $2 \mathrm{D}$ en dominio tiempo (TABLA 2), analizadas por medio del software libre SeiSee 2.2. La descripción de facies sísmicas tiene en cuenta el grado de contraste de impedancia entre reflectores, relacionado a la amplitud de la óndula (fuerte, débil), continuidad lateral y relación entre los reflectores.

TABLA 2. Coordenadas geográficas e información de líneas sísmicas 2D (sistema de coordenadas proyectadas MAGNA Colombia Bogotá) usadas en el presente estudio.

\begin{tabular}{|c|c|c|c|c|c|c|c|}
\hline \multirow{2}{*}{ ID } & \multirow{2}{*}{ NOMBRE } & \multirow{2}{*}{ LONGITUD } & \multicolumn{4}{|c|}{ COORDENADAS } & \multirow{2}{*}{ REFERENCIA } \\
\hline & & & X INICIO & Y INICIO & X FINAL & Y FINAL & \\
\hline 1 & BHP-CPE5-2010-01 & 13.479 .066 & 1.505 .629 & 1.041 .615 & 1.505 .517 & 1.041 .616 & suministrado por la $\mathrm{ANH}$ \\
\hline 2 & RO-1980-01 & 79.741 .498 & 1.472 .406 & 1.076 .085 & 1.461 .092 & 1.084 .022 & suministrado por la $\mathrm{ANH}$ \\
\hline 3 & RO-1981-01N & 78.519 .242 & 1.367 .950 & 1.162 .644 & 1.426 .618 & 1.110 .461 & suministrado por la $\mathrm{ANH}$ \\
\hline 4 & CE-1985-2040 & 43.032 .559 & 1.471 .504 & 1.202 .063 & 1.505 .892 & 1.176 .075 & suministrado por la $\mathrm{ANH}$ \\
\hline 5 & SAL-2007-04 & 46.828 .629 & 1.357 .993 & 1.017 .863 & 1.386 .183 & 1.007 .170 & suministrado por la $\mathrm{ANH}$ \\
\hline 6 & HP-1981-06 & 84.586 .243 & 1.241 .421 & 905.820 & 1.317 .784 & 936.884 & suministrado por la $\mathrm{ANH}$ \\
\hline 7 & RM-1991-446W & 52.442 .619 & 1.103 .471 & 895.498 & 1.155 .902 & 895.362 & suministrado por la $\mathrm{ANH}$ \\
\hline 8 & C-1980-1285 & 71.182 .062 & 1.196 .778 & 1.044 .821 & 1.251 .827 & 999.696 & suministrado por la $\mathrm{ANH}$ \\
\hline
\end{tabular}

\section{Mapas de isócoros y de tasas de acumulación}

Se elaboraron mapas de espesores verticales en pozos (mapas isócoros) de las unidades litológicas correspondientes a los miembros informales $\mathrm{C} 1 \mathrm{y}$ C2 de la Formación Carbonera y de la Formación León con el propósito de conocer los cambios geométricos regionales de la cuenca durante la depositación, producto de la actividad tectónica, clima y eustasia. Estos espesores junto con el tiempo de acumulación estimado para el pozo SALTARIN 1A, permiten la elaboración de mapas que cuantifican el comportamiento de las tasas de acumulación durante la depositación de las incursiones marinas en el sector distal de los Llanos. Tomando como datos de entrada la interpretación de superficies de correlación de pozos presentados en Bayona et al. (2009a-2009b). La variación espacial de espesores y tasas de acumulación se modelaron por procesos aleatorios con autocorrección espacial, que prevén valores en las ubicaciones sin información de pozos, mediante la utilización de la herramienta de análisis geoestadístico (Kriging) en el software ArcGis 10.3. 


\section{ANÁLISIS BIOESTRATIGRAFICO, SEDIMENTOLÓGICO Y ESTRATIGRÁFICO}

A continuación, se hace un resumen de la revisión del análisis sedimentológico y estratigráfico secuencial para el pozo SALTARIN 1A (FIGURA 3) y de la sección de Medina (FIGURA 4), integrando los resultados de bioestratigrafía.

\section{Análisis sedimentológico y estratigráfico secuencial para el pozo SALTARIN 1A}

Este pozo perforó el segmento superior de la Formación
Carbonera (intervalos informales $\mathrm{C} 3, \mathrm{C} 2$ y $\mathrm{C} 1$ ). E1 intervalo de interés en este estudio es el $\mathrm{C} 2$, el cual presenta un espesor de 46 metros, correspondiente a una sucesión lodosa, laminada y localmente bioperturbada, en las cuales, las litofacies varían entre lodolitas claras macizas a lodolitas verdosas laminadas paralelas, irregulares y/o discontinuas. En esta unidad se interpretan cuatro secuencias estratigráficas de cuarto orden. El mayor porcentaje de índice de salinidad es del $95 \%$ y la superficie de máxima inundación del Mioceno temprano se presenta a una profundidad de 621 metros (base de la biozona T-13, $17.4 \mathrm{my}$ ), correspondiendo a un sistema de centro de lago profundo con aguas marinas (FIGURA 3).

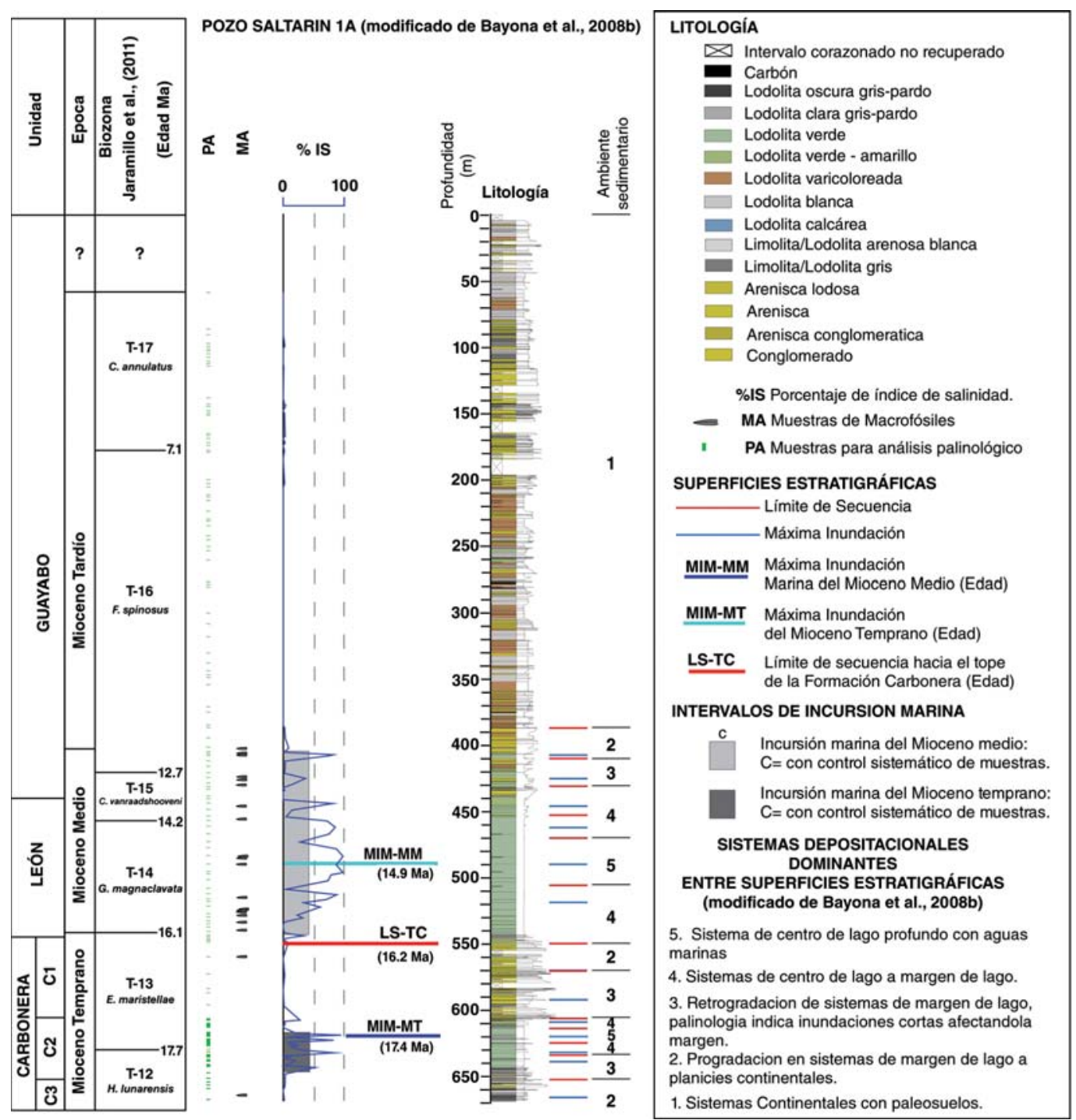

FIGURA 3. Columna estratigráfica generalizada del pozo SALTARIN 1A (modificado de Bayona et al., 2008b), y análisis bioestratigráfico presentados en Jaramillo et al. (en revisión). la zonación bioestratigráfica sigue a Jaramillo et al. (2011). Se indican los límites de secuencia y superficies máximas de inundación, así como los sistemas depositacionales entre estas superficies estratigráficas. La integración de los resultados del porcentaje de palinomorfos marinos con la anterior información permite determinar los intervalos de incursión y las superficies que registran los eventos de máxima inundación marina del Mioceno. 
A partir de este evento de máxima inundación, el sistema prograda coincidiendo con el intervalo informal C1. Este intervalo, de 61 metros de espesor, incluye areniscas conglomeráticas macizas gradando a areniscas de granulometría medias a finas (Bayona et al., 2008b). Este intervalo se localiza en sistemas de margen de lago llegando a planicies continentales, las capas más espesas al tope del intervalo presentan $0 \%$ de índice de salinidad localizando el límite de secuencia (superficie de máxima progradación) FIGURA 3.

El segmento inferior a medio de la Formación León presenta un espesor de 75 metros, correspondiente a una sucesión de litofacies homogéneas que consisten principalmente de lodolita verdosa laminada paralela a masiva. En este segmento se identificaron 2 secuencias de cuarto orden, las cuales incluyen la incursión marina del Mioceno medio. Para el intervalo de $552 \mathrm{~m}$ a $534 \mathrm{~m}$, el sistema de sedimentación retrograda de margen de lago a centro de lago somero, presentando índices de salinidad no superiores al 3\% (predominio de aguas frescas). Posterior a este intervalo, el sistema se inunda y se incrementa el índice de salinidad, retrogradando a sistemas de centro de lago profundo con predominio de aguas marinas, en donde la máxima superficie de inundación se registra a una profundidad de 490 metros (tope de la biozona T-14, 14.9 my), la cual corresponde al intervalo con mayor influencia marina (porcentajes de índice de salinidad entre $73 \%$ a 98\%) (FIGURA 3).

Otras tres secuencias fueron identificadas en el segmento superior $(30 \mathrm{~m})$; sin embargo estas, en comparación con el segmento anterior, hacen parte del sistema de progradación que incluye la base de la Formación Guayabo.

Los análisis palinológicos indican una edad Mioceno temprano para la Formación Carbonera (biozonas T-12 a T-13; 18.5 my a 16 my), mientras la Formacion León es del Mioceno medio (biozonas T-14 a la base del T-15; 16 my a 13.5 my). La Formación Guayabo es de edad Mioceno medio - tardío con registro de las biozonas T-15 a T-17 (13.5 my a 5.5 my), aunque para los 60 metros más superiores no fue posible determinar una edad (FIGURA 3).

\section{Análisis sedimentológico y estratigráfico secuencial para la sección de Medina}

El intervalo informal C2 de la Formación Carbonera presenta un espesor de 194 metros depositados en ambientes estuarinos, correspondiente a una sucesión lodosa, localmente bioperturbada, con intercalaciones arenosas. Las sucesiones son estrato-crecientes en las cuales las litofacies varían entre lodolitas grises oscuras monótonas y areniscas de grano fino a medio (Parra et al., 2010). En este nivel se logran identificar tres secuencias estratigráficas de cuarto orden (FIGURA 4). El mayor porcentaje de influencia marina ( $42 \%$ de índice de salinidad) se presenta al nivel estratigráfico de 2872 metros. Este intervalo se caracteriza por un abundante contenido fosilífero, dominado por gasterópodos Sheppardiconcha, y Bivalvos Anodontites, Mytilopsis, indicadores de ambientes fluviolacustres a marginales (Gomez et al., 2009). Al nivel estratigráfico de 2713 metros se presenta un nivel muy delgado $(7-15 \mathrm{~mm})$ de especies de la familia Arcidae, indicador de ambientes marinos (Gomez et al., 2009). Este último intervalo lo interpretamos como el registro de la máxima inundación marina del Mioceno temprano, correspondiendo a un sistema de centro de lago profundo con aguas marinas.

Suprayaciendo este intervalo C2, el sistema prograda a depósitos fluviales trenzados, correspondiente a las intercalaciones arenosas de la Formación Carbonera (miembro informal C1) de 112 metros de espesor (Parra et al., 2010). Este intervalo se caracteriza por la presencia de areniscas lodosas, y areniscas de grano fino a medio granocrecientes, con laminación ondulosa, lenticular e inclinada planar, intercalada con lodolitas grises y varicoloreadas (Parra et al., 2010). Los estratos del intervalo $\mathrm{C} 1$ representan la retrogradación del sistema de margen de lago a planicies continentales (FIGURA 4).

La Formación León, presenta un espesor de 640 metros, correspondientes a un conjunto de lodolitas negras a grises oscuras localmente bioperturbadas e intercalaciones de capas fosilíferas con bivalvos diseminados y concreciones calcáreas con macrofósiles depositadas en ambientes estuarinos (Parra et al., 2010). En este segmento se identificaron 4 secuencias de cuarto orden, e incluye la incursión marina del Mioceno medio. Los índices de salinidad son inferiores al $24 \%$, en donde la máxima superficie de inundación se registra a una profundidad de 1433 metros, correspondiendo a un sistema de centro de lago profundo con aguas marinas (FIGURA 4).

Los análisis palinológicos indican una edad Mioceno temprano para la Formación Carbonera (biozonas T-12 a T-13; 19.13 my a 16.16 my), mientras la Formación León es del Mioceno medio (biozonas T-14 a T-16; 16.16 my a 11.88 my). La Formación Guayabo es de edad Mioceno superior - Plioceno con registro de las biozonas T-16 a T-18 (11.88 my a 1.07 my) (FIGURA 4). 


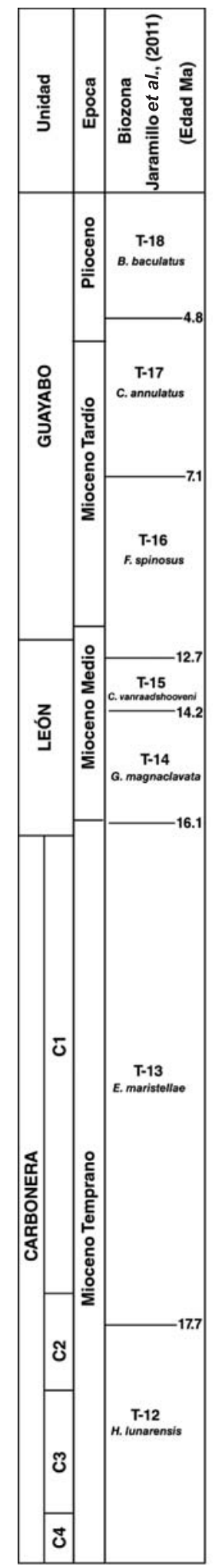

SECCION DE MEDINA (modificado de Parra et al., 2010)

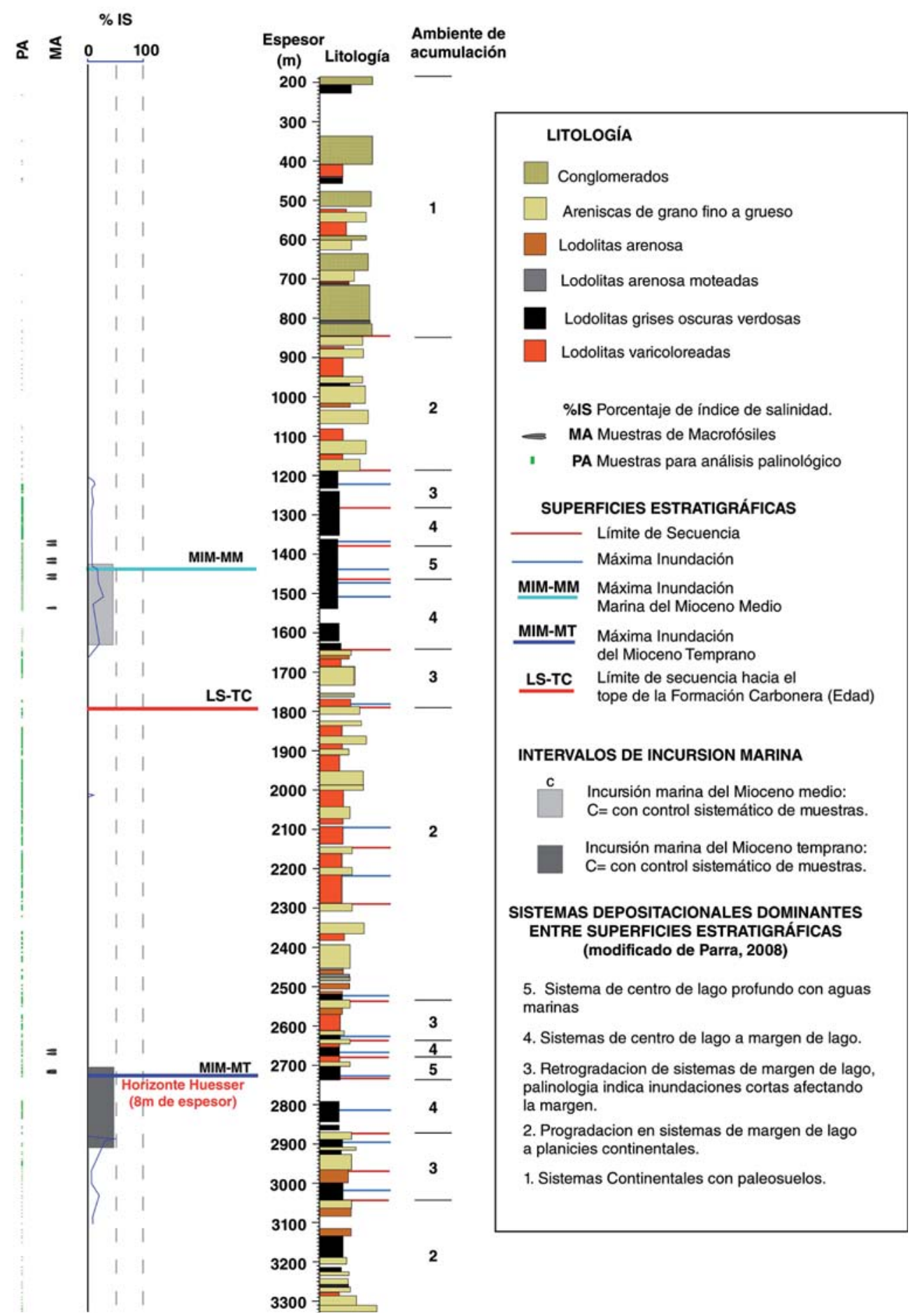

FIGURA 4. Columna estratigráfica generalizada de la sección de MEDINA (modificado de Parra et al., 2010), El análisis bioestratigráfico y la zonación bioestratigráfica es tomada de Jaramillo et al. (2011). Se indican los límites de secuencia y superficies máximas de inundación, así como los sistemas deposicionales entre estas superficies estratigráficas. La integración de los resultados del porcentaje de palinomorfos marinos con la anterior información permite determinar los intervalos de incursión y las superficies que registran los eventos de máxima inundación marina del Mioceno. 


\section{ESTRATIGRAFÍA Y CORRELACIÓN POZOS PROFUNDOS}

Las superficies cronoestratigráficas descritas para el pozo SALTARIN 1A y para la sección de Medina se han correlacionado entre sí, identificando superficies similares en seis (6) pozos profundos (TABLA 1) que unen estas dos secciones y con interpretaciones previas de bioestratigrafía y análisis secuencial ya publicadas (Bayona et al., 2007; Bayona et al., 2009a; Bayona et al., 2009b). Las electrofacies indican que las incursiones marinas se localizan en secciones cilíndricas con altos valores API que suprayacen la tendencia de campana producto de la retrogradación de los sistemas e infrayacen la tendencia de embudo producto de la progradación de los sistemas de acumulación (FIGURA 5).

\section{Pozo D}

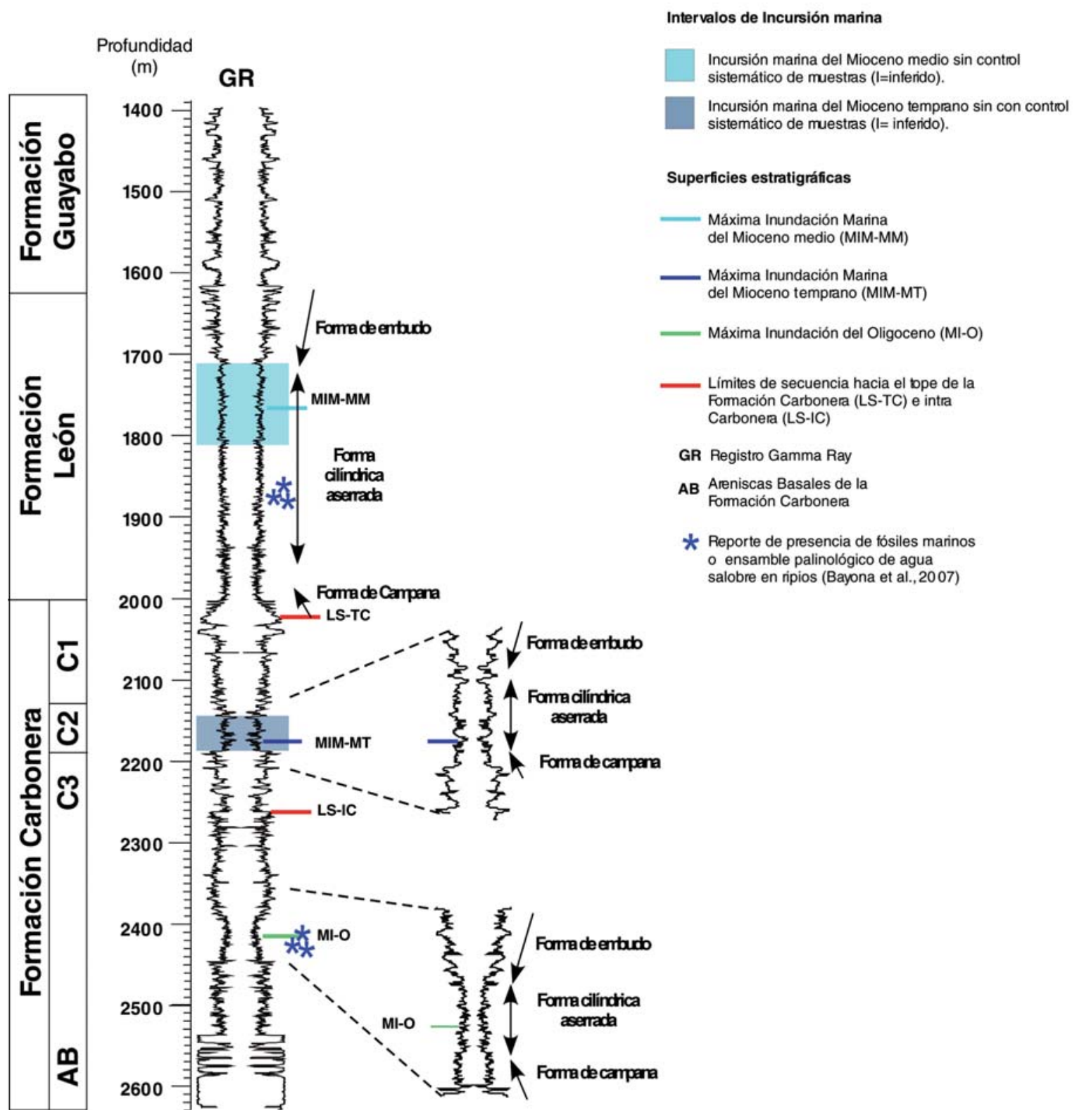

FIGURA 5. Análisis de electrofacies para la identificación de superficies estratigráficas de máxima inundación marina del Mioceno y los límites de secuencia. Los intervalos de incursiones marinas se localizan en secciones cilíndricas con altos valores de GR, dominadas por lodolitas y acumuladas en llanuras de inundación, llanuras costeras o fondos de lago. Estos intervalos suprayacen la tendencia de campana producto de la retrogradación de los sistemas de acumulación e infrayacen la tendencia de embudo producto de la progradación de los sistemas de acumulación. 
Para este análisis también se considera los cambios globales del nivel base (nivel del mar), presentados en Hardenbol et al. (1998) y la geometría de cuenca de antepaís producto de los controles tectónicos, dados por la subsidencia flexural y topografía dinámica (Bayona et al., 2009a; Bayona et al., 2009b; Mora et al., 2015) y aporte de sedimentos (Bayona et al., 2007; Campos and Mann, 2015; Roddaz et al., 2010). En una cuenca tipo antepaís continental, se presentan cambios laterales en la geometría y procedencia de las areniscas producto de la relación entre la tasa de generación de espacio de acomodación/ aporte de sedimentos (Bayona et al., 2009b). La mayor subsidencia se localiza hacia el foredeep, sector en el cual se presenta mayor espacio de acomodación, favoreciendo el registro espeso del aporte clástico producto de la denudación del frente orogénico. El espacio de acomodación disminuye hacia el forebulge, caracterizado por presentar menor subsidencia por el levantamiento flexural. Si el forebulge está emergido, este segmento corresponde al área fuente de los depósitos arenosos que se acumulan en el sector más distal del foredeep (FIGURA 6A); por el contrario, si el forebulge está por debajo del nivel base, ya sea por subsidencia producto de topografía dinámica, por aumento del nivel de base global o por sobrellenado de la cuenca por alto aporte sedimentario, este segmento junto con la parte distal del foredeep se convierte en la zona axial de los sistemas de acumulación (Reyes-Harker et al., 2015) procedentes del orógeno al occidente de las rocas expuestas sobre el escudo de la Guyana al oriente (FIGURA 6B), e inclusive de altos intracuenca expuesto en el Mioceno medio-tardío al sur en el Arco del Vaupés (Bayona et al., 2008a; Bayona et al., 2008b).

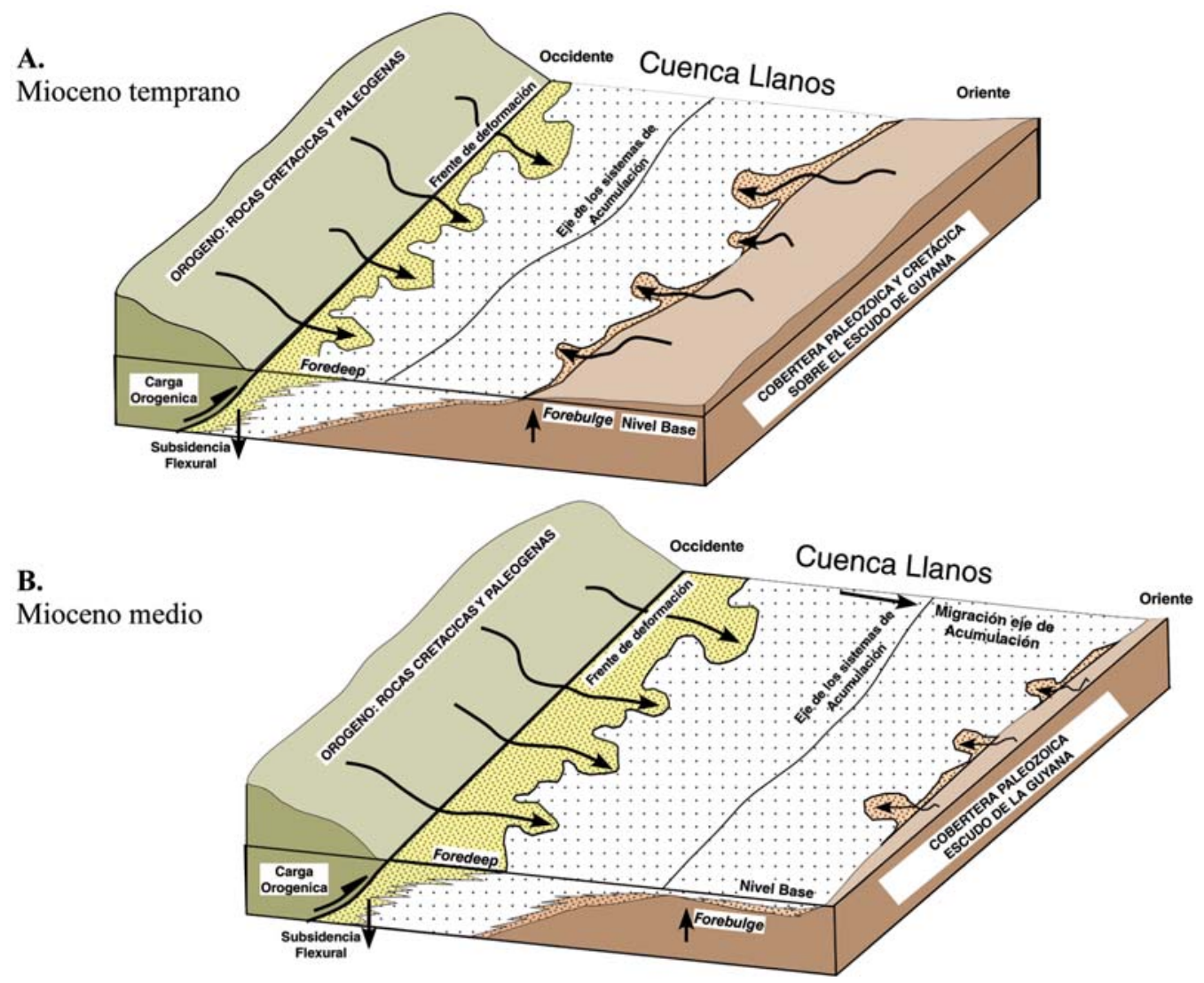

FIGURA 6. Bloque diagrama ilustrando las áreas fuente que limitan la cuenca de antepaís de los Llanos orientales y la posición relativa del forebulge con respecto al cambio del nivel de base regional. A. En el Mioceno temprano el nivel de base por debajo del forebulge limitando la extensión de la cuenca. B. En el Mioceno medio el nivel base asciende (ya sea por eustasia y/ó aumento de la subsidencia por aporte de sedimentos) y el forebulge migra al oriente y queda cubierto por la acumulación; como resultado, la extensión de la cuenca se amplia. Adicionalmente migra al oriente tanto el eje de acumulación, donde se registra la acumulación lodosa, como el sector de acumulación arenosa oriental (Areniscas basales de la Formación Carbonera, Bayona et al. 2007).

Las anteriores consideraciones permiten identificar tres superficies de máxima inundación separados por dos límites de secuencia (superficie de máxima progradación) hacia la parte media y el tope de la
Formación Carbonera (FIGURA 7). La superficie más antigua corresponde a la máxima inundación del Oligoceno (MIM-O; tope de la biozona T-11), la cual se restringe a la zona axial que separa los dos sistemas 
de acumulación descritos para el foredeep. El límite de secuencia intra-Carbonera (LS-IC; biozona T-12) suprayace el apilamiento agradacional a progradacional, el cual se observa en los pozos B a H (FIGURA 7). En la sección de Medina se documenta el apilamiento de litofacies lodosas continentales que progradan a litofacies arenosas fluviales, en el pozo SALTARIN 1A se infiere que esta sucesión no este registrada por ser el segmento expuesto del forebulge durante el Oligoceno (Bayona et al., 2009b).

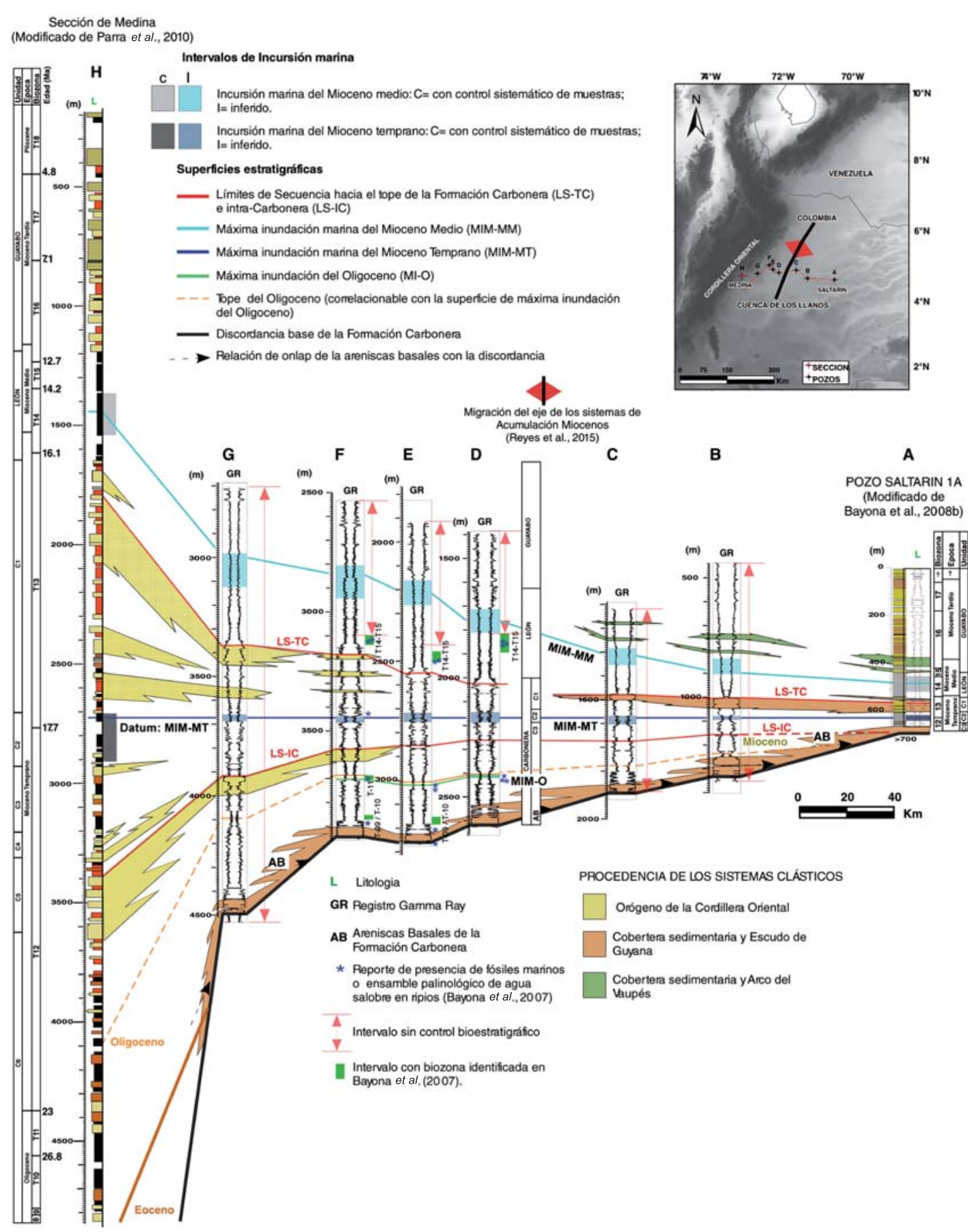

FIGURA 7. Correlación estratigráfica desde el pozo SALTARIN 1A (sector distal) hasta la sección de Medina (sector proximal) donde se identifican dos superficies de máxima inundación marina del Mioceno, separadas por dos límites de secuencia hacia la parte media y el tope de la Formación Carbonera. La presente correlación usa los registros Gamma Ray (GR), descripción de litologías, presencia de fósiles marinos de aguas salobres en ripios y biozonación reportados en Bayona et al. (2007). La zonación bioestratigráfica para la sección de Medina y el pozo Saltarín-1A sigue a Jaramillo et al. (2011). 
Posterior a este evento de progradación, se registra el intervalo de la incursión marina del Mioceno temprano. La superficie de máxima inundación marina del Mioceno temprano (MIM-MT; base de biozona T-13) se ha seleccionado como Datum de la correlación entre los pozos por corresponder a un intervalo más delgado, fácilmente reconocible en registros GR y con buen contraste de impedancias en las facies sísmicas (como se describe en la siguiente sección). El límite de secuencia hacia el tope de la Formación Carbonera (LS-TC; tope de la biozona $\mathrm{T}-13$ ) infrayace el apilamiento retrogradacional que va desde los sistemas continentales, a los de margen y centro de lago. Esta retrogradación es abrupta en el sector distal de la cuenca mientras al occidente del eje de acumulación el cambio es más gradual (FIGURA 7). La superficie de máxima inundación marina del Mioceno medio (MIM-MM; tope de la biozona T-14) se localiza en los sistemas de centro de lago profundo con indicadores marinos.

\section{ANÁLISIS DE LA INFORMACIÓN SÍSMICA}

Este procedimiento permite obtener un contexto regional del comportamiento de los dos intervalos de incursión marina y de los estratos adyacentes desde el sector distal hasta el sector proximal de la cuenca Llanos Orientales. La facies sísmica de la Formación León y el miembro informal C2 de la Formación Carbonera se caracteriza por presentar reflectores con baja amplitud de picos y valles generando un aspecto "sordo" por la ausencia de contraste de impedancia en las litologías lodosas homogéneas; sin embargo, hacia los bordes de la cuenca se incrementa la presencia de reflectores (FIGURA 8). Los reflectores del miembro arenoso $\mathrm{C} 3$ de la Formación Carbonera se caracterizan por ser de alta amplitud de picos y valles, son discontinuos y no paralelos con respecto a la facies sísmica del miembro C2 (FIGURA 8). Los reflectores correspondientes a la unidad arenosa $\mathrm{C} 1$ tienen una alta amplitud de picos y valles, con extensión lateral de varias decenas de kilómetros y siguen un paralelismo con respecto a la facies sísmica del miembro C2 (FIGURA 8). La facies sísmica correspondiente a las intercalaciones arenosas y lodosas a la base de la Formación Guayabo presenta reflectores con alta amplitud de picos y valles debido al contraste de litologías (FIGURA 8), pero a medida que se siguen al oriente pierden amplitud y se pierde su expresión en la facies sísmica "sorda" de la Formación León. El miembro inferior de la Formación Guayabo se identifica por la presencia de altas amplitudes de sus picos y valles, mayor continuidad lateral de los reflectores a la base y mayor discontinuidad hacia el tope.
Debido al carácter "sordo" que caracteriza a los dos intervalos de incursión marina del Mioceno, no es posible identificar sísmicamente la superficie de máxima inundación marina para cada intervalo. Por consiguiente, esta identificación debe valerse de un buen amarre con la información de pozo, además de una alta densidad de muestreo para análisis palinológicos. Sin embargo, debido al reducido espesor de la incursión marina del Mioceno temprano se puede inferir el trazo de la superficie de máxima inundación marina del Mioceno temprano (MIM-MT) y utilizarla como datum de correlación a nivel regional (FIGURA 8).

\section{MAPA DE ESPESORES Y TASAS DE ACUMULACIÓN}

Mapas isócoros (espesores verticales en pozos) permiten conocer los cambios geométricos de la cuenca durante la depositación, producto de la actividad tectónica/ clima/eustasia. En este trabajo comparamos el espesor vertical de: (1) los miembros $\mathrm{C} 2$ y $\mathrm{C} 1$, unidades que incluyen el intervalo de la incursión marina del Mioceno temprano (FIGURA 9A), (2) con respecto al espesor de la Formación León (FIGURA 9C), unidad litológica que incluye el intervalo de la incursión marina del Mioceno medio. La elaboración de mapas isócoros y mapas de tasas de acumulación en rocas del Mioceno tienen en cuenta que la geometría de muy bajo ángulo de buzamiento $\left(<4^{\circ}\right)$ en el segmento más proximal de la cuenca no varía en más del $1 \%$ el valor espesor real de las unidades, mientras en el sector distal las capas están horizontales.

El mapa del Mioceno temprano (FIGURA 9A) ilustra la posición del depocentro directamente relacionados a la orientación del frente de deformación. La actividad orogénica se localizaría a lo largo del todo el frente de deformación, principalmente al sur de la sección de Medina. Hacia la parte distal de la cuenca, la tendencia de los espesores presenta un aumento homogéneo hacia el norte siguiendo la geometría de la curva del frente de la deformación entre la Cordillera Oriental de Colombia y los Andes de Mérida.

El mapa de la Formación León, unidad que incluye el evento de inundación del Mioceno medio, presenta los mayores espesores de esta unidad litológica en dos depocentros (FIGURA 9C). El primer depocentro se localiza hacia el frente de deformación y su geometría sugiere que la deformación se concentró al norte de la sección de Medina; sin embargo, el depocentro no sigue la tendencia de la curva del frente de la deformación en su sector más norte y en los Andes de Mérida. El segundo depocentro se localiza hacia el sector distalcentral de la cuenca y tiene una geometría concentrica. 


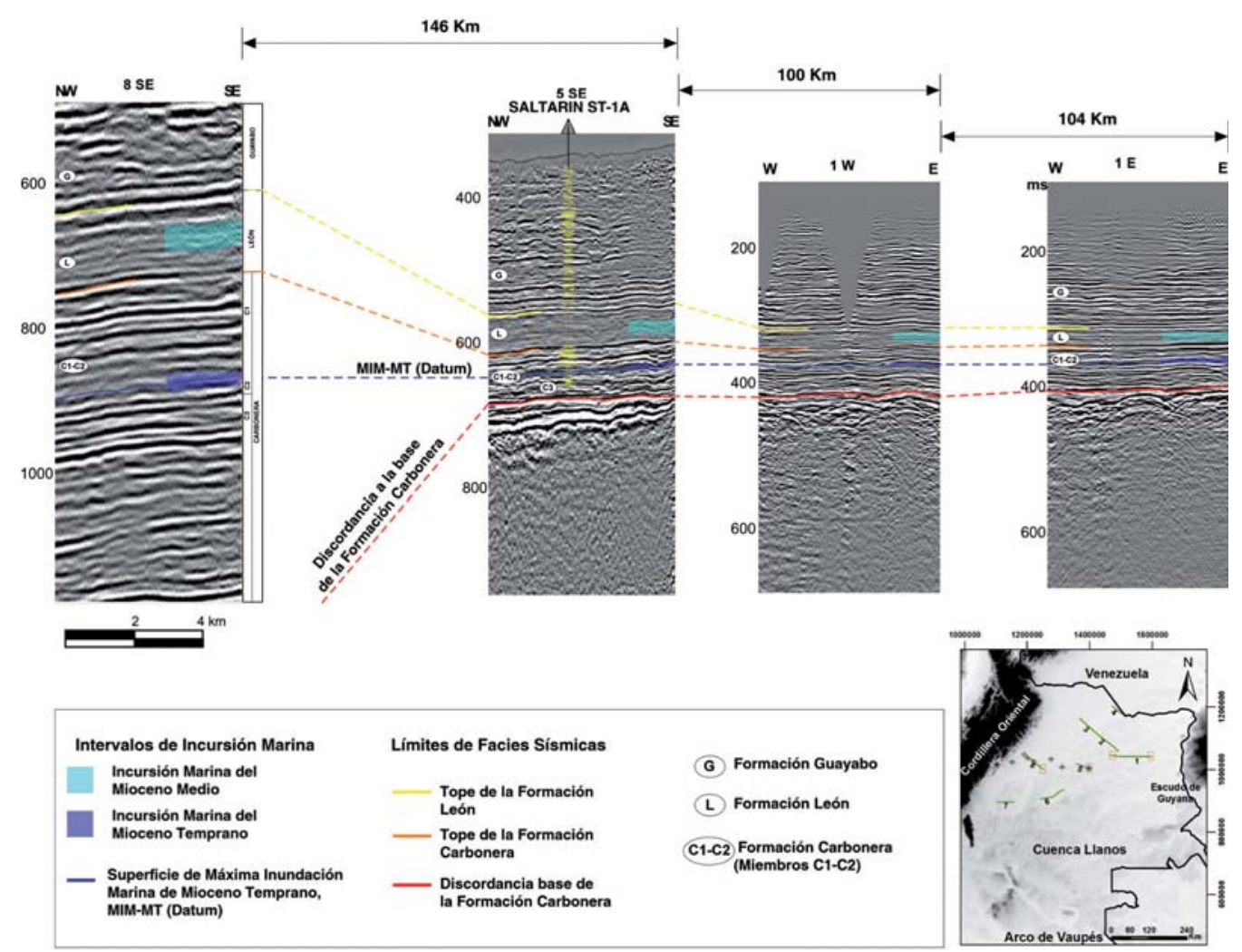

FIGURA 8. Perfiles sísmicos en la cuenca de los Llanos (segmentos corresponden a un extremo de la línea, ver mapa inferior) con la interpretación de las facies sísmicas correspondientes a las dos incursiones marinas del Mioceno temprano y medio. El datum corresponde a la superficie de máxima inundación marina del Mioceno temprano, debido a que esta superficie es fácilmente reconocible en toda la cuenca.

Aunque estos mapas tienen como referencia contactos de unidades litológicas, estos contactos pueden tener un significado temporal válido en el segmento distal de la cuenca, en donde la geometría estratigráfica es más tabular (oriente del pozo D en la FIGURA 7). Para cuantificar el comportamiento de las tasas de acumulación en el sector distal, se toma como referencia el espesor de cada intervalo y el tiempo de acumulación estimado para el pozo SALTARIN 1A (FIGURAS 9B y 9D). En general, hay un incremento regional en el Mioceno medio de la tasa de acumulación. Usando como referencia los valores del pozo B (más cercano al depocentro concéntrico), los espesores compactados correspondientes a la inundación marina del Mioceno temprano presentan espesores de 95 metros depositados en 2 millones de años. Para la inundación marina del Mioceno medio los espesores alcanzan 325 metros depositados en 3 millones de años. A partir de las anteriores observaciones se estima que la tasa de acumulación durante es Mioceno medio es de $108 \mathrm{~m} / \mathrm{my}$, y duplica la tasa de acumulación de $47 \mathrm{~m} / \mathrm{my}$ calculada para el Mioceno temprano. En la siguiente sección daremos una explicación a este cambio de incremento en la subsidencia total en este sector distal de la cuenca.

\section{DISCUSIÓN DE RESULTADOS}

\section{Validez de las superficies estratigráficas de correlación (cronoestratigráficas)}

La identificación de los diferentes tipos de superficies estratigráficas tiene en cuenta parámetros como litología, registros eléctricos, sísmica, palinología, los cuales permiten clasificar las superficies estratigráficas en dos grandes grupos (FIGURA 10). El primer grupo corresponde a las superficies de topes de unidades litológicas (e.g., tope de la Formación León) cuya identificación está condicionada al contraste entre litologías, representadas en (1) respuesta de los registros eléctricos, y (2) contrastes de impedancia en líneas sísmicas. La formación de estas superficies se encuentra controlada por la subsidencia flexural, el suministro de sedimentos de las áreas fuente en una cuenca de antepaís y la eustasia (e.g., FIGURA 6). Por consiguiente, al comparar el tiempo de generación de estas superficies litológicas es diferente (diacrónico) hacia los bordes de la cuenca (donde están las áreas fuente) con respecto al depocentro de la cuenca. El segundo grupo corresponde a las superficies estratigráficas con valor 
cronoestratigráfico (sincrónicas). La identificación de estas superficies incorporan adicionalmente análisis palinológicos y estratigráficos secuenciales, no se restringe a los controles tectónicos locales (FIGURA 10), y son el reflejo de cambios globales del nivel base.

Una de las superficies estratigráficas con valor cronoestratigráfico corresponde a la superficie de máxima inundación marina del Mioceno temprano (MIM-MT). Esta superficie se logra reconocer regionalmente en la cuenca de los Llanos debido a: (1) el dominio de litologías finas homogéneas y alto valores API (FIGURA 7); (2) su limitado espesor permite documentar la superficie de máxima inundación marina con una menor cantidad de muestras de palinología ; (3) la baja amplitud de sus reflectores sísmicos en este nivel de geometría tabular que separa los suprayacentes reflectores de alta amplitud con geometría plana paralela continua (miembro $\mathrm{C} 1$ ), mientras los reflectores de las unidades infrayacentes (miembro C3) son de mayor amplitud, discontinuos y no paralelos (FIGURA 8). Estos argumentos hacen reconocible esta superficie tanto en sísmica como en registros eléctricos; por consiguiente, consideramos que esta es la superficie de correlación regional que se puede identificar en toda la cuenca de los Llanos.

FORMACIÓN CARBONERA (miembros informales C1 + C2; Mioceno temprano)
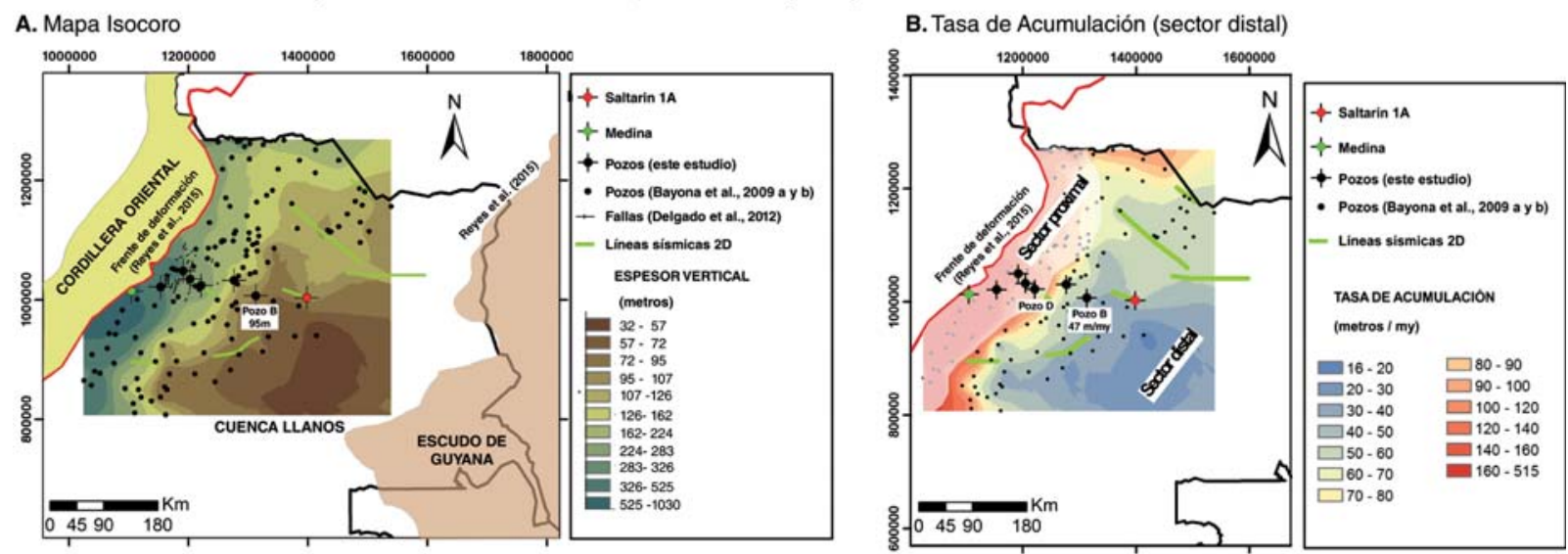

\section{FORMACIÓN LEÓN (Mioceno medio)}

C. Mapa Isocoro

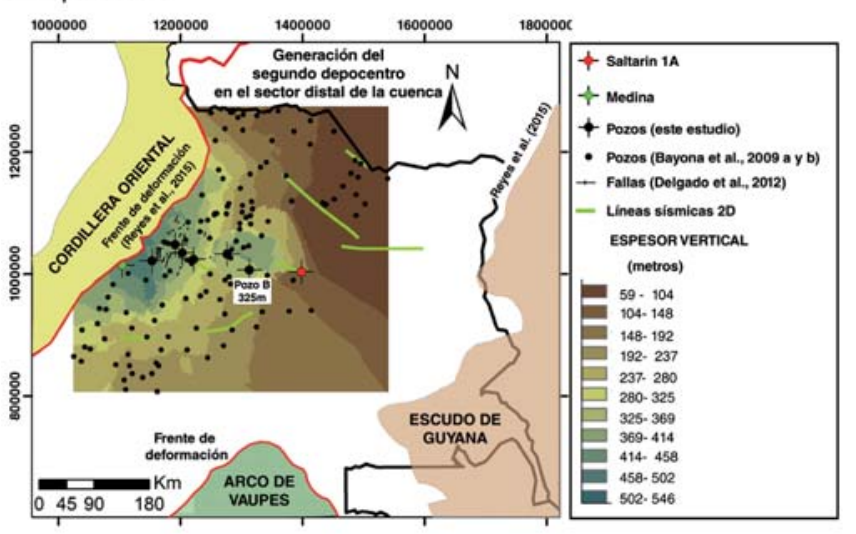

D. Tasa de Acumulación (sector distal)

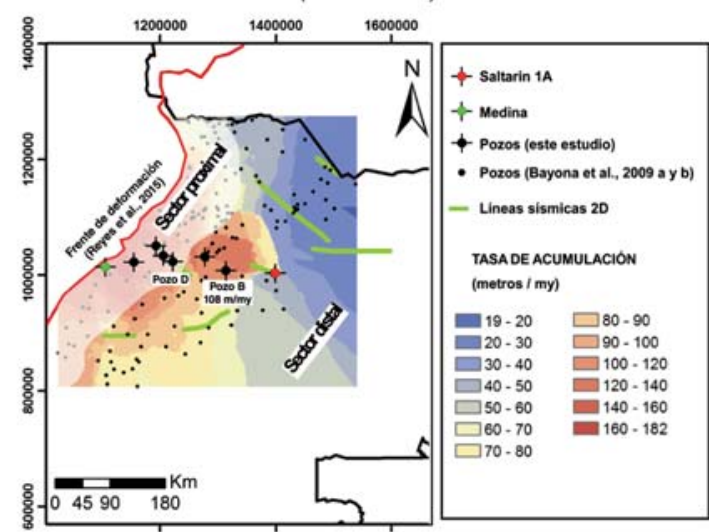

FIGURA 9. A. Mapa isocoro equivalente al espesor de los miembros informales $\mathrm{C} 2+\mathrm{C} 1$ de la Formación Carbonera. B. Tasas de acumulación con énfasis para el sector distal de la cuenca de las unidades $\mathrm{C} 2+\mathrm{C} 1$. C. Mapa isocoro equivalente al espesor de la Formación León. D. Tasas de acumulación con énfasis para el sector distal de la cuenca de la Formación León. Ver discusión en el texto.

Otra superficie estratigráfica con valor cronoestratigráfico corresponde a la superficie de máxima inundación marina del Mioceno medio (MIM-MM). Esta superficie se encuentra en un intervalo que posee facies sísmicas fácilmente reconocibles a nivel regional, pero la delimitación de esta superficie se dificulta por: (1) un gran espesor; (2) pobre resolución sísmica y homogeneidad de los 
registros eléctricos con alto Gamma Ray, y (3) escasez de análisis palinológicos. Estas dos transgresiones marinas regionales también han sido documentadas en la cuenca Oriental de Venezuela, en donde la sedimentación marina es relativamente continua y registrada en los depósitos turbidíticos de la Formación Carapita del Mioceno temprano-medio (Parnaud et al., 1995a; Parnaud et al., 1995b). Lo anterior sugiere que las ingresiones marinas registradas en los Llanos ocurren a lo largo de la cuenca Oriental de Venezuela, ya que el inicio del levantamiento de los Andes de Mérida desde el Oligoceno (Bermúdez et al., 2010) interrumpe la comunicación marina con la cuenca de Maracaibo.
La superficie de máxima inundación del Oligoceno (MI-O) presenta pobre extensión regional de la influencia salobre en los pozos analizados (FIGURA 7); sin embargo, los análisis palinológicos en ripios de un pozo en el piedemonte indican un mayor registro de la influencia salobre (pozo C-BA; en Bayona et al., 2007). Los depósitos asociados a esta superficie cambian lateralmente hacia el piedemonte a depósitos marginales con un incremento de espesor de litofacies finas y hacia el sector distal de los Llanos cambia a las areniscas basales continentales y el punto de no registro en el cratón. Sin embargo, se requiere de un análisis bioestratigráfico sistemático en núcleos para corroborar la presencia de esta superficie estratigráfica.
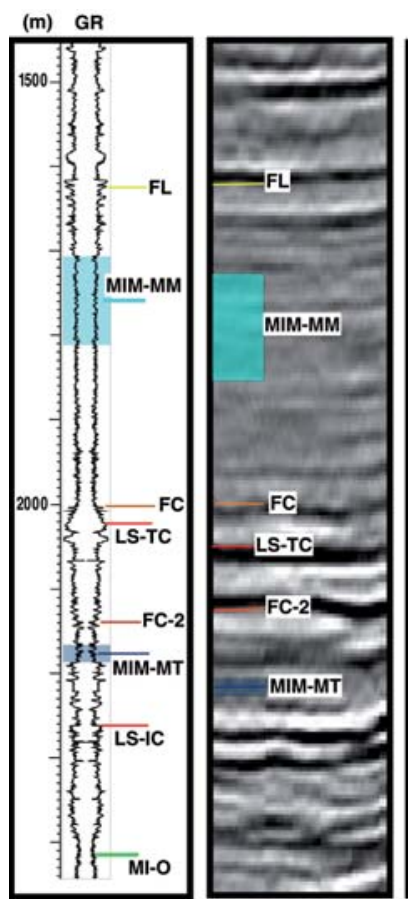

\begin{tabular}{|c|c|c|c|c|c|}
\hline $\begin{array}{l}\text { Superficies de } \\
\text { Correlación }\end{array}$ & $\begin{array}{c}\text { Litología \& } \\
\text { Registros } \\
\text { eléctricos (GR) }\end{array}$ & Sismica 2D & Palinología & $\begin{array}{l}\text { Diacronismo/ } \\
\text { Sincronismo }\end{array}$ & $\begin{array}{l}\text { Estratigrafia de } \\
\text { secuencias }\end{array}$ \\
\hline $\begin{array}{l}\text { (FL) Tope de la } \\
\text { Formación León }\end{array}$ & Transicional & Transicional & NA & Diacrónico & NA \\
\hline $\begin{array}{l}\text { (MiM-MM) Máxima } \\
\text { inundación marina } \\
\text { del Mioceno Medio }\end{array}$ & No detectable & No detectable & $\begin{array}{l}\text { Detectable } \\
\text { (Requiere } \\
\text { alto muestreo) }\end{array}$ & Sincrónico & $\begin{array}{c}\text { Detectable } \\
\text { (con palinologia) }\end{array}$ \\
\hline $\begin{array}{l}\text { (FC)Tope de la } \\
\text { Formación Carbonera }\end{array}$ & $\begin{array}{c}\text { Transicional a } \\
\text { Neto }\end{array}$ & Neto & NA & Diacrónico & NA \\
\hline \begin{tabular}{|l|} 
(LS-TC / LS-IC) Limites \\
deSecuencia hacia el tope \\
de la Fm. Carbonera e \\
intra-Carbonera
\end{tabular} & Neto & $\begin{array}{l}\text { detectable si hay } \\
\text { contraste de } \\
\text { Iitologias }\end{array}$ & $\begin{array}{l}\text { Pobre recobro en } \\
\text { facies arenosas }\end{array}$ & Sincrónico & $\begin{array}{l}\text { Detectable } \\
\text { (con registros) }\end{array}$ \\
\hline \begin{tabular}{|l}
$($ FC-2) Tope del \\
nivel intormal C2 \\
(Fomación \\
Carbonera)
\end{tabular} & Transicional & No detectable & NA & Diacrónico & NA \\
\hline $\begin{array}{l}\text { (MIM-MT) Máxima } \\
\text { inundación marina del } \\
\text { Mioceno Temprano }\end{array}$ & Neto & $\begin{array}{l}\text { Facies sordas } \\
\text { del miembro C2 }\end{array}$ & $\begin{array}{l}\text { Detectable } \\
\text { (Requiere } \\
\text { bajo Muestreo) }\end{array}$ & Sincrónico & $\begin{array}{c}\text { Detectable } \\
\text { (Con registros y } \\
\text { palinologia) }\end{array}$ \\
\hline $\begin{array}{l}\text { (MI-O) Máxima } \\
\text { inundación del } \\
\text { Oligoceno }\end{array}$ & No detectable & No detectable & $\begin{array}{c}\text { Detectable } \\
\text { (Requiere } \\
\text { alto Muestreo) }\end{array}$ & $\begin{array}{l}\text { Diacronico } \\
\text { (Pobre extension } \\
\text { regional) }\end{array}$ & $\begin{array}{c}\text { Detectable } \\
\text { (con palinologia) }\end{array}$ \\
\hline
\end{tabular}

FIGURA 10. Criterios para la identificación de las superficies de correlación, a partir de los diferentes tipos de análisis realizados en este estudio. La superficie de Máxima inundación marina del Mioceno temprano (MIM-MT) se destaca como la de mayor facilidad de identificación y confiabilidad en su trazo a nivel regional.

El límite de secuencia hacia el tope de la Formación Carbonera (LS-TC, ó superficie de máxima progradación hacia el tope de la biozona T13), presenta una tendencia heterogénea de los patrones de apilamiento y litologías debido a las variaciones en las tasas de acomodación y aporte de sedimentos en la cuenca de antepaís (FIGURA 6). En la zona axial a distal de la cuenca se observa una variación abrupta del paso de una secuencia progradacional, dominada por sistemas clástico arenoso, procedentes del escudo de la Guyana a una secuencia retrogradacional dominados por litologías finas (FIGURA 7, pozos A-C). Hacia la zona proximal, donde la acumulación es más espesa y aumenta el aporte de los clásticos del frente orogénico, el patrón de apilamiento retrogradacional es gradual, en el cual se presentan varias intercalaciones entre litologías arenosas y lodosas (FIGURA 7, pozos D-H). Procesos similares se pueden asociar a los estratos que suprayacen e infrayacen el límite de secuencia intra-Formación Carbonera (LS-IC).

Factores que controlan la generación de las superficies de máxima inundación Marina del Mioceno

Dadas las anteriores consideraciones, las dos superficies de máxima inundación marina del Mioceno temprano 
y medio, junto con los límites de secuencia, son las de mayor relevancia como superficies de correlación regional en la cuenca de los Llanos (FIGURA 7). El ejercicio de aplanamiento de la información sísmica usando la superficie de máxima inundación marina del Mioceno medio, ejercicio válido ya que el cambio de relieve interno (topografía vs batimetría) en la cuenca es relativamente mínimo (decena de metros), permitirá en futuros estudios con sísmica 3D documentar la migración lateral de los canales fluviales y entrampamiento estratigráfico de hidrocarburos en la cuenca de los Llanos Orientales en Colombia.

Los mapas de espesor vertical presentan un depocentro hacia el sector proximal de la cuenca y adyacente al frente de deformación de la Cordillera Oriental (FIGURAS 9A y 9C). Estas zonas de mayor espesor en la zona proximal del piedemonte implican una carga de enterramiento para la roca fuente del Cretácico y Paleógenas durante el Mioceno medio y tardío. Las rocas de litologías finas del Mioceno son el sello regional intraformacional, y se podrían considerar como reservorio para sistemas no convencionales de hidrocarburos.

Los factores que pueden explicar el incremento en la tasa de acumulación en la cuenca de los Llanos (FIGURAS 9B y 9D), manteniendo constante la litología lodosa durante los intervalos de incursión marina (FIGURA 11), se clasifican en dos grandes grupos:

El primer grupo corresponde a los factores relacionados a la deformación y aporte de sedimentos que afectan a escala de cuenca.

(1) Las tasas de acortamiento del flanco oriental de la Cordillera Oriental entre 20 a 9 Ma es de $0.84 \mathrm{~mm} /$ año aumentando a $3.30 \mathrm{~mm} /$ año entre 9 a $5 \mathrm{Ma}$ (Mora et al., 2015); Si asumimos un incremento en la tasa de acortamiento entre el Mioceno temprano y Mioceno medio se originaria una mayor carga tectónica que favorecería el incremento del espacio de acumulación y el avance hacia el cratón de la subsidencia flexural durante el Mioceno medio (Bayona et al., 2009a; 2009b).

(2) Levantamientos intracuenca en el sector sur de los Llanos (Arco de Vaupés) genera un frente de deformación $\mathrm{y}$ un aumento en el suministro de sedimentos hacia el sector sur-distal de la cuenca (Bayona et al., 2008a; Bayona et al., 2007; Mora et al., 2010; Reyes-Harker et al., 2015). Esta deformación afectaría la geometría de la subsidencia flexural y explicaría la geometría concéntrica del depocentro de las litologías lodosas de la Formación León.
(3) El incremento de la subsidencia total de la cuenca para el Mioceno temprano y medio (Bayona et al., 2009a) que permite la acumulación de los depósitos lodosos en los cuales se registran los eventos de inundación marina se refleja por un incremento simultáneo en el aporte de sedimentos procedentes de la denudación de los sistemas clásticos del Orógeno de la Cordillera oriental (Parra et al., 2010), de la cobertera sedimentaria asociada al Escudo de Guyana y de la cobertera sedimentaria asociada al Arco del Vaupés (Bayona et al., 2008b) y la generación de espacio de acomodación representado por el incremento de la tasa de acomodación en la parte distal de la cuenca de los Llanos entre el Mioceno temprano ( $47 \mathrm{~m} / \mathrm{my}$ ) al Mioceno medio $(108 \mathrm{~m} / \mathrm{my})$. La acumulación de los sistemas clásticos presenta una migración lateral, depositando las granulometrías gruesas hacia el sector proximal donde se presenta mayor espacio de acumulación, mientras las granulometrías finas ocupan posiciones axiales en el sistema de acumulación y hacia el sector distal de los llanos, donde el espacio de acomodación aumenta con el tiempo por el aumento de la subsidencia total de la cuenca resultado de la combinación de flexura, carga de sedimentos y eusatasia.

El segundo grupo representa factores más regionales a la escala de cuenca, y consideramos que deben tenerse en cuenta en el análisis de la subsidencia y relleno sedimentario.

(4) Variaciones en la tasa máxima de cambio del nivel del mar representadas en la variación del nivel base de primer orden (Hardenbol et al., 1998) muestra una mayor tasa durante la incursión marina del Mioceno medio $(\sim 3.8 \mathrm{~m} / \mathrm{my})$ en comparación con la tasa durante la incursión del Mioceno inferior $(\sim 2.2 \mathrm{~m} / \mathrm{my})$.

(5) Las variaciones climáticas a nivel global, presentadas en (Zachos et al., 2001) nos indican que posterior al enfriamiento del Oligoceno, siguió una fase cálida, la cual alcanzó su punto máximo en el óptimo climático del Mioceno medio ( $\sim 15 \mathrm{my})$ y a la cual le prosigue un enfriamiento gradual. Modelos climáticos indican que las tasas de precipitación aumentaron durante el Mioceno (Jeffery et al., 2011) lo cual pudo favorecer la denudación del orógeno, cratón y altos intracuenca; originando una mayor tasa de suministro de sedimentos y el aumento de la subsidencia por carga de sedimentos.

(6) La topografía dinámica desempeña un papel relevante en la subsidencia regional en las cuencas del norte de Suramérica. Los modelos geodinámicos de topografía dinámica presentados en Shephard et al. (2010) indican una influencia a escala continental $(\sim 3.000 \mathrm{~km})$ en el 
incremento en la subsidencia del Mioceno temprano y medio (10 a 20 my), alcanzando una subsidencia superior a los 200 metros. Sin embargo, la escala en la que se desarrolló el modelo impide explicar y diferenciar los incrementos en las tasas de acumulación durante el Mioceno temprano y medio en la cuenca Llanos $(\sim 300$ $\mathrm{km})$. Además, las tasas de convergencia presentadas por Somoza and Ghidella (2005) muestran una convergencia constante durante el Mioceno temprano (140 mm/yr) y disminuye gradualmente durante el Mioceno medio (125 mm/yr) sin cambio en el buzamiento de la placa (Echeverri et al., 2015).

\begin{tabular}{|c|c|c|c|}
\hline Factores & $\begin{array}{l}\text { Mioceno } \\
\text { temprano }\end{array}$ & $\begin{array}{l}\text { Mioceno } \\
\text { medio }\end{array}$ & Referencias \\
\hline $\begin{array}{l}\text { 1. Tasa de acortamiento }(T A)+ \\
\text { subsidencia flexural }\end{array}$ & $\mathrm{TA}=0.84 \mathrm{~mm} / \mathrm{año}$ & $\mathrm{TA}=0.84 \mathrm{~mm} / \mathrm{año}$ & $\begin{array}{l}\text { Bayona et al., (2009a-b) } \\
\text { Mora et al. (2015) }\end{array}$ \\
\hline 2. Levantamiento intracuenca & & Arco de Vaupés & $\begin{array}{l}\text { Bayona et al., (2008b) } \\
\text { Mora et al (2010) }\end{array}$ \\
\hline $\begin{array}{l}\text { 3. Aporte de sedimentos }+ \text { subsidencia } \\
\text { por carga de sedimentos }\end{array}$ & Orógeno + craton & $\begin{array}{l}\text { Orógeno }+ \text { craton }+ \\
\text { altos intracuenca }\end{array}$ & Este estudio \\
\hline 4. Cambio global del nivel del mar & $\sim 2.2 \mathrm{~m} / \mathrm{my}$ & $\sim 3.8 \mathrm{~m} / \mathrm{my}$ & Hardenbol et al. (1998) \\
\hline 5. Clima & $\begin{array}{l}\text { menor } \\
\text { temperatura }\end{array}$ & $\begin{array}{l}\text { óptimo } \\
\text { climático }\end{array}$ & Zachos et al. (2001) \\
\hline 6. Topografía dinámica y subducción & $\sim 300 \mathrm{~m}$ & $\sim 300 \mathrm{~m}$ & Shephard et al. (2010) \\
\hline
\end{tabular}
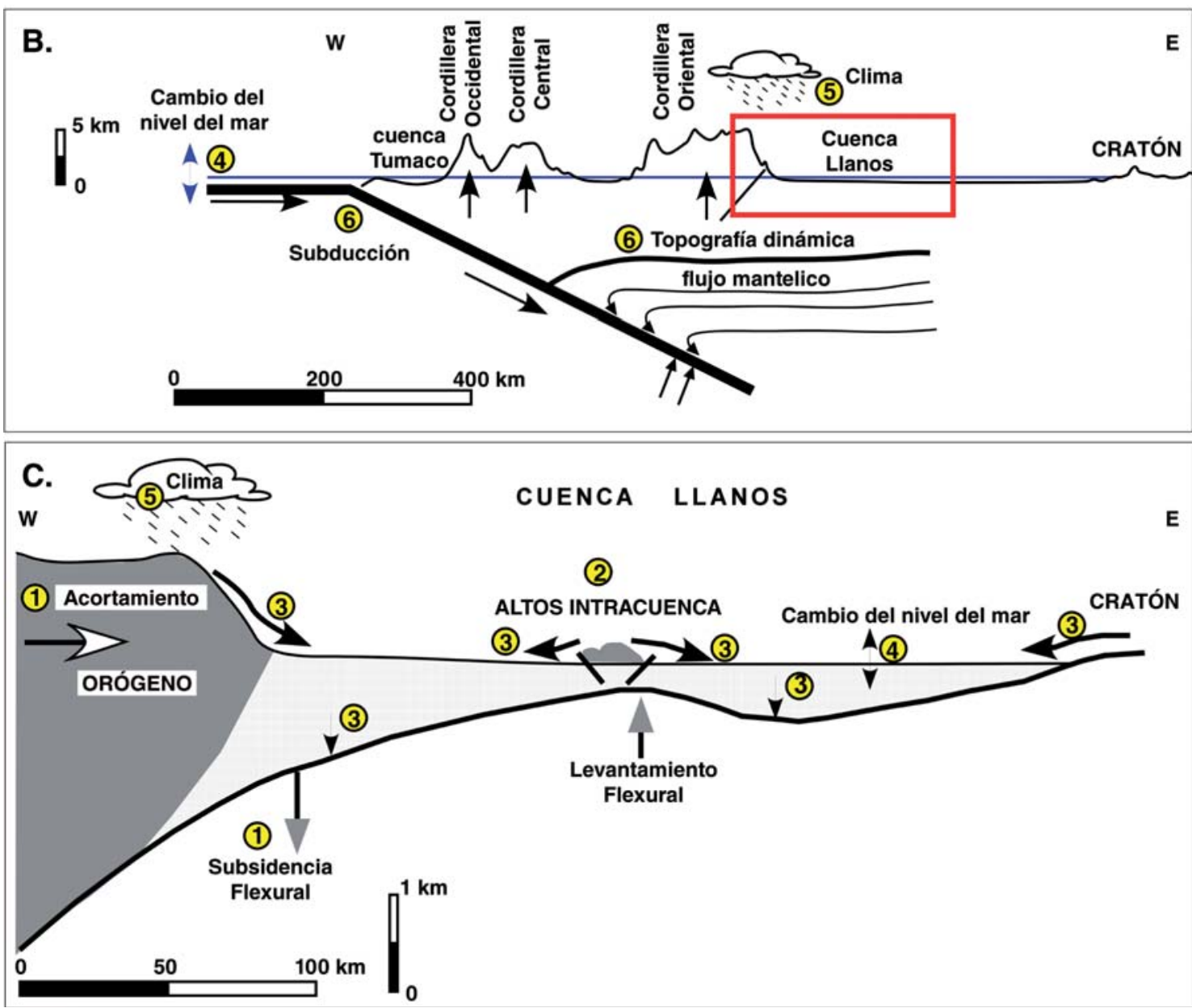

FIGURA 11. A. Resumen de los principales factores que pueden explicar el incremento en la tasa de acumulación en la cuenca de los Llanos, manteniendo constante la litología lodosa durante los intervalos de incursión marina. B. Esquema ilustrando los factores relacionados a la deformación y aporte de sedimentos que afectan a escala de cuenca. C. Esquema ilustrando los factores más regionales a la escala de cuenca. 


\section{CONCLUSIONES}

La integración de los análisis sedimentológicos, palinológico, estratigráfico secuencial y la interpretación sísmica y de pozos permite identificar dos intervalos de incursión marina en la cuenca de los Llanos Orientales de Colombia, y para cada evento ubicar la superficie de máxima inundación marina. La primera superficie corresponde a la superficie máxima de inundación marina del Mioceno temprano (base de la biozona T-13), localizada en el miembro informal C2 de la Formación Carbonera; y la segunda superficie corresponde a la máxima inundación marina del Mioceno medio (tope de la biozona T-14) localizada hacia el sector medio a superior de la Formación León. Estas dos superficies de máxima inundación marina del Mioceno tienen alta relevancia como superficies de correlación debido a su comportamiento sincrónico y extensión regional, las cuales cubren desde el sector proximal hasta el sector distal de la cuenca de antepaís. Sin embargo, la superficie de máxima inundación marina del Mioceno temprano es más fácil de identificar debido a su reducido espesor de litologías finas, fácil identificación en registros eléctricos y en la sísmica, y su identificación mediante análisis de estratigrafía secuencial. La máxima superficie de inundación del Mioceno medio no es detectable en registros eléctricos ni en sísmica 2D debido a su gran espesor. Estos eventos de inundación marina están separados por límites de secuencia del Mioceno temprano localizados hacia la parte media y al tope de la Formación Carbonera, pero no son de fácil seguimiento con la información sísmica debido a que no presentan contrastes fuertes de litologías con espesores significativos para generar facies sísmicas identificables. Otra superficie de correlación estratigráfica es la máxima inundación del Oligoceno (MI-O); sin embargo, estratos asociados a esta superficie presentan solo influencia salobre y su continuidad lateral se determina por el análisis estratigráfico secuencial en los pozos analizados. Los procesos deposicionales durante el Mioceno temprano y medio que afectan la cuenca de los Llanos, están controlados por la combinación de eventos tectónicos relacionados a la subsidencia flexural que se origina por el el incremento en el acortamiento y consecuente levantamiento de la Cordillera Oriental, levantamientos intracuenca relacionados al Arco de Vaupés y cambios globales en el nivel del mar. El incremento de la tasa de acomodación en la parte distal de la cuenca de los Llanos entre el Mioceno temprano (47 m/my) al Mioceno medio $(108 \mathrm{~m} / \mathrm{my})$ se explica por el incremento tanto del aporte de sedimentos como de la generación de espacio de acomodación. En contraste, los depocentros localizados al frente de la zona de deformación orogénica delimitan zonas de mayor espesor e implican una carga de enterramiento para la roca fuente del Cretácico y Paleógeno durante el Mioceno medio y tardío. Posibles trampas estratigráficas pueden visualizarse mejor al aplanar la información sísmica usando las superficies de máxima inundación marina.

\section{AGRADECIMIENTOS}

Agradecemos a COLCIENCIAS por la financiación a partir del programa Jóvenes Investigadores e innovadores 2014, así mismo por los aportes otorgados a la Corporación Geológica ARES en el plan de fortalecimiento institucional a centros de investigación. A la Agencia Nacional de Hidrocarburos (ANH) por su colaboración y disposición de la información sísmica y de pozos utilizada en el desarrollo del presente proyecto. A HOCOL S.A. por el acceso al pozo y al muestreo detallado en el 2008 para realizar estos análisis (tesis de maestría de Ingrid Romero) y a los evaluadores anónimos quienes aportaron importantes comentarios que permitieron mejorar el contenido y presentación de este manuscrito.

\section{REFERENCIAS}

Bayona, G., Jaramillo, C., Rueda, M., Reyes-Harker, A., and Torres, V. 2007. Paleocene-middle Miocene flexural-margin migration of the nonmarine Llanos foreland basin of Colombia. CT\&F Ciencia, Tecnología y Futuro, 3(3): 141-160.

Bayona, G., Cortes, M., Jaramillo, C., Ojeda, G., Aristizabal, J., and Reyes-Harker, A. 2008a. An integrated analysis of an orogen-sedimentary basin pair: Latest Cretaceous-Cenozoic evolution of the linked Eastern Cordillera orogen and the Llanos foreland basin of Colombia. Geological Society of America Bulletin, 120(9-10): 1171-1197.

Bayona, G., Valencia, A., Mora, A., Rueda, M., Ortiz, J., and Montenegro, O. 2008b. Estratigrafía y procedencia de las rocas del Mioceno en la parte distal de la cuenca de antepais de los Llanos de Colombia. Geología Colombiana, 33: 23-46.

Bayona, G., Valencia, A., de Armas, M., Guerrero, J., Gómez, E., Leyva, I., Villamarin, P., and Mora, A. 2009a. Oligocene - Miocene filling of the distal Llanos Basin of Colombia: Interaction of flexural subsidence, intraplate faulting and dynamic topography models. AAPG International Conference and Exhibition, Rio de Janeiro, Brasil. 
Bayona, G., Villamarin, P., Mora, A., Ojeda, G., Cortes, M., Valencia, A., Mahecha, H., and Torres, V. 2009b. Exploratory implications of forebulge geometry and migration in the Llanos Basin. X Simposio Bolivariano de Cuencas Subandinas, Cartagena, Colombia.

Beaumont, C. 1981. Foreland basins. Geophysical Journal of the Royal Astronomical Society, 65(2): 291-329.

Bermúdez, M.A., Kohn, B., Van der Beek, P.A., Bernet, M., O'Sullivan, P., and Shagam, R. 2010. Spatial and temporal patterns of exhumation across the Venezuelan Andes: Implications for Cenozoic Caribbean geodynamics. Tectonics, 29(5): 2-21.

Boonstra, M., Ramos, M.I.F., Lammertsma, E.I., Antoine, P.O., and Hoorn, C. 2015. Marine connections of Amazonia: Evidence from foraminifera and dinoflagellate cysts (early to middle Miocene, Colombia/Peru). Palaeogeography, Palaeoclimatology, Palaeoecology, 417: 176-194.

Campos, H., and Mann, P. 2015. Tectonostratigraphic evolution of the Northern Llanos Foreland Basin of Colombia and implications for its hydrocarbon potential. In: Bartolini, C., and Mann, P. (eds.). Petroleum geology and potential of the Colombian Caribbean Margin. AAPG Memoir, 108: 517-456.

Catuneanu, O. 2004. Retroarc foreland systemsevolution through time. Journal of African Earth Sciences, 38(3): 225-242.

Catuneanu, O., Abreu, V., Bhattacharya, J.P., Blum, M.D., Dalrymple, R.W., Eriksson, P.G., Fielding, C.R., Fisher, W.L., Galloway, W.E., Gibling, M.R., Giles, K.A., Holbrook, J.M., Jordan, R., Kendall, C.G.S.C., Macurda, B., Martinsen, O.J., Miall, A.D., Neal, J.E., Nummedal, D., Pomar, L., Posamentier, H.W., Pratt, B.R., Sarg, J.F., Shanley, K.W., Steel, R.J., Strasser, A., Tucker, M.E., and Winker, C. 2009. Towards the standardization of sequence stratigraphy. Earth-Science Reviews, 92(1-2): 1-33.

Cooper, M.A., Addison, F.T., Alvarez, R., Coral, M., Graham, R.H., Hayward, A.B., Howe, S., Martinez, J., Naar, J., Peñas, R., Pulham, A. J., and Taborda, A. 1995. Basin development and tectonic history of the Llanos basin, Eastern Cordillera, and Middle Magdalena Valley, Colombia. AAPG Bulletin, 79(10): 1421-1443.

DeCelles, P.G., and Giles, K.A. 1996. Foreland basin systems. Basin Research, 8(2): 105-123.
Delgado, A., Mora, A., and Reyes-Harker, A. 2012. Deformation partitioning in the Llanos foreland basin during the Cenozoic and its correlation with mountain building in the hinterland. Journal of South American Earth Sciences, 39: 228-244.

Echeverri, S., Cardona, A., Pardo, A., Monsalve, G., Valencia, V.A., Borrero, C., Rosero, S., and López, S. 2015. Regional provenance from southwestern Colombia fore-arc and intra-arc basins: Implications for Middle to Late Miocene orogeny in the Northern Andes. Terra Nova, 27(5): 356-363.

Giles, K.A., and Dickinson, W.R. 1995. The interplay of eustasy and lithospheric flexure in forming stratigraphic sequences in Foreland setting: An example from the Antler Foreland, Nevada and Utah. In: Stratigraphic evolution of Foreland Basins. Dorobek, S.L., and Ross, G.M. (Eds.). Volume 52: Tulsa, SEPM.

Gómez, A., Jaramillo, C., Parra, M., and Mora, A. 2009. Huesser horizon: A lake and a marine incursion in Northwestern South America during early Miocene. Palaios, 24(4): 199-210.

Gómez, J., Montes, N.E., Novia, A., and Diederix, H. 2015. Mapa Geológico de Colombia 2015, Escala 1:1000000: Servicio Geológico Colombiano, 2 hojas.

Hackley, P., Urbani, F., Karlsen, A.W., and Garrity, C. 2006. Mapa Geológico de Venezuela, Escala 1:750000: USGS OPEN-FILE REPORT 2006-1109.

Hardenbol, J., Thierry, J., Farley, M.B., Jacquin, T., de Graciansky, P.C., and Vail, P. 1998. Mesozoic and Cenozoic sequence chronostratigraphic framework of European basin. In: Graciansky, P. C. (Ed.). Mesozoic and Cenozoic Sequence Stratigraphy of European Basins. Vol. 60, SEPM Special Publicantion, p. 3-13.

Hermeston, S., and Nemcok, M. 2013. Thick-skin orogen - foreland interactions and their controlling factors, Northern Andes of Colombia. Geological Society of London, Special Publication, 377: 443-471.

Hoorn, C., Guerrero, J., Sarmiento, G.A., and Lorente, M.A. 1995. Andean tectonics as a cause for changing drainage patterns in Miocene northern South America. Geology, 23(3): 237-240.

Hoorn, C., Paxton, C., Crampton, W., Burgess, P., Marshall, L., Lundberg, J., Rasanen, M., and Linna, A. 1996. Miocene deposits in the Amazonian Foreland Basin. Science, 273(5271): 122-125. 
Jaramillo, C., Romero, I.C., D’Apolito, C., Bayona, G., Duarte, E., Louwye, S., Escobar, J., Luque, J., CarrilloBriceño, J.D., Zapata, V., Mora, J.A., Schouten, S., Zavada, M., Harrington, G., Ortiz, J., and Wesseling, F.P. 2016. Miocene flooding events of Western Amazonia. En revisión - Science Advances.

Jaramillo, C., Rueda, M., and Torres, V. 2011. A palynological zonation for the Cenozoic of the Llanos and Llanos Foothills of Colombia. Palynology, 35(1): 46-84.

Jeffery, M.L., Poulsen, C.J., and Ehlers, T.A. 2011. Impacts of Cenozoic global cooling, surface uplift, and an inland seaway on South American paleoclimate and precipitation $\delta 180$. Geological Society of America Bulletin, 124(3-4): 335-351.

Montaño, P.C., Nova, G., Bayona, G., Mahecha, H., Ayala, C., Jaramillo, C., and De la Parra, F. 2016. Análisis de secuencias y procedencia en sucesiones sedimentarias de grano fino: Un ejemplo de la Formación Umir y base de la Formación Lisama, en el sector de Simacota (Santander, Colombia). Boletín de Geología, 38(1): 51-72.

Mora, A., Baby, P., Roddaz, M., Parra, M., Brusset, S., Hermoza, W., and Espurt, N. 2010. Tectonic history of the Andes and sub-Andean zones: Implications for the development of the Amazon drainage basin. In: Hoorn, C., and Wesselingh, F.P. (Ed.). Amazonia, landscape and species evolution: A look into the past. Oxford, WileyBlackwell Publishing, pp. 38-60.

Mora, A., Casallas, W., Ketcham, R.A., Gomez, D., Parra, M., Namson, J., Stockli, D., Almendral, A., Robles, W., and Ghorbal, B. 2015. Kinematic restoration of contractional basement structures using thermokinematic model: A key tool for petroleum system modeling. AAPG Bulletin, 99(8): 1575-1598.

Moreno-López, M.C., and Escalona, A. 2015. Precambian-Pleistocene tectono-stratigraphic evolution of the southern Llanos Basin, Colombia. AAPG Bulletin, 99(8): 1473-1501.

Parnaud, F., Gou, Y., Pascual, J.C., Capello, M.A., Truskowski, I., and Passalacqua, H. 1995a. Stratigraphic synthesis of western Venezuela. In: Tankard, A.J., Suárez, R., and Welsink, H.J. (Eds.). Petroleum basins of South America. AAPG Memoir 62, 681-698.

Parnaud, F., Gou, Y., Pascual, J.C., Truskowski, I., Gallango, O., Passalacqua, H., and Roure, F. 1995 b.
Petroleum geology of the central part of the Eastern Venezuela basin. In: Tankard, A.J., Suárez, R., and Welsink, H.J. (Eds.). Petroleum basins of South America, AAPG Memoir 62, 741-756.

Parra, M., Mora, A., Jaramillo, C., Strecker, M.R., Sobel, E.R., Quiroz, L.I., Rueda, M., and Torres, V. 2009a. Orogenic wedge advance in the northern Andes: Evidence from the Oligocene-Miocene sedimentary record of the Medina Basin, Eastern Cordillera, Colombia. Geological Society of America Bulletin, 121(5-6): 780-800.

Parra, M., Mora, A., Sobel, E., Strecker, M.R., and González, R. 2009b. Episodic orogenic front migration in the northern Andes: Constraints from low-temperature thermochronology in the Eastern Cordillera, Colombia. Tectonics, 28(4): 1-27.

Parra, M., Mora, A., Jaramillo, C., Torres, V., Zeilinger, G., and Strecker, M. R. 2010. Tectonic controls on Cenozoic foreland basin development in the north-eastern Andes, Colombia. Basin Research, 22(6): 874-903.

Pemberton, S.G., and MacEachern, J.A. 1995. The sequence stratigraphic significance of trace fossils: Examples from the Cretaceous foreland basin of Alberta, Canada. In: Van Wagoner, J.C., and Bertram, G.T. (Ed.). Sequence stratigraphy of foreland basin deposits. Vol. 64: AAPG, Tulsa, pp. 429 - 475.

Reyes-Harker, A., Ruiz-Valdivieso., C.F., Mora, A., Ramirez-Arias, J.C., Rodriguez, G., De la Parra, F., Caballero, V., Parra, M., Moreno, N., Horton, B.K., Saylor, J.E., Silva, A., Valencia, V., Stockli, D., and Blanco, V. 2015. Cenozoic paleogeography of the Andean foreland and retroarc hinterland of Colombia. AAPG Bulletin, 99(8): 1407-1453.

Roddaz, M., Hermoza, W., Mora, A., Baby, P., Parra, M., Christophoul, F., Brusset, S., and Espurt, N. 2010. Cenozoic sedimentary evolution of the Amazonian foreland basin system. In: Hoorn, C., and Wesselingh, F. (Eds.). Amazonia, landscape and species evolution: A look into the past. Blackwell Publishing, pp. 61-88.

Romero, I. C. 2014. Palynological evidence for the paleoenvironmental history of the Miocene Llanos Basin, Eastern Colombia. M.Sc. Thesis, Seton Hall University, South Orange, 75p.

Santos, C., Jaramillo, C., Bayona, G., Rueda, M., and Torres, V. 2008. Late Eocene marine incursion in north-western South America. Palaeogeography, Palaeoclimatology, Palaeoecology, 264(1-2): 140-146. 
Sarmiento, L. F. 2011. Geology and hydrocarbon potential Llanos basin. In: Cediel, F., and Ojeda, G. Y. (Eds.). Petroleum geology of Colombia. Volume 9: Medellin, ANH, Fondo editorial Universidad Eafit, p. 17-184.

Schwans, P. 1995. Control on sequence stacking and fluvial to shallow-marine architecture in a foreland basin. In: Van Wagoner, J.C., and Bertram, G.T. (eds.). Sequence stratigraphy of foreland basin deposits. Vol. 64: Tulsa, AAPG, pp. 55-101.

Shephard, G.E., Müller, R.D., Liu, L., and Gurnis, M. 2010. Miocene drainage reversal of the Amazon River driven by plate-mantle interaction. Nature Geoscience, 3: 870-875.

Somoza, R., and Ghidella, M.E. 2005. Convergencia en el margen occidental de América del Sur durante el Cenozoico: Subducción de las placas de Nazca, Farallón y Aluk. Revista de la Asociación Geológica Argentina, 60(4): 797-809.

Torrado, L., Mann, P., and Bhattacharya, J. 2014. Application of seismic attributes and spectral decomposition for reservoir characterization of a complex fluvial system: Case study of the Carbonera Formation, Llanos foreland basin, Colombia. Geophysics, 79(5): B221-B230.

Traverse, A. 2007. Paleopalynology. Springer. Dordrecht.

Van Wagoner, J.C. 1995. Overview of sequence stratigraphy of foreland basin deposits: Terminology, summary of papers, and glossary of sequence stratigraphy. In: Van Wagoner, J.C., and Bertram, G.T. (Eds.). Sequence stratigraphy of foreland basin deposits. Vol. 64: Tulsa, AAPG, p. ix - xxi.

Villamil, T. 2003. Regional hydrocarbon systems of Colombia and western Venezuela: Their origin, potential, and exploration. In: Bartolini, C., Buffler, R.T., and Blickwede, J. (eds.). The Circum-Gulf of Mexico and the Caribbean: Hydrocarbon habitats, basin formation, and plate tectonics. AAPG Memoir, 79, pp. 697-734.

Zachos, J., Pagani, M., Sloan, L., Thomas, E., and Billups, K. 2001. Trends, Rhythms, and Aberrations in Global Climate 65 Ma to Present. Science, 292(5517): 686-693.

Trabajo recibido: agosto 10 de 2016

Trabajo aceptado: noviembre 28 de 2016

Manuscrito publicado en internet: diciembre 14 de 2016 